MATHEMATICS OF COMPUTATION

Volume 81, Number 277, January 2012, Pages 533-554

S 0025-5718(2011)02509-3

Article electronically published on July 1, 2011

\title{
THE IMAGINARY ABELIAN NUMBER FIELDS OF 2-POWER DEGREES WITH IDEAL CLASS GROUPS OF EXPONENT $\leq 2$
}

\author{
JEOUNG-HWAN AHN AND SOUN-HI KWON
}

\begin{abstract}
In this paper, assuming the Generalized Riemann Hypothesis, we determine all imaginary abelian number fields $N$ of 2-power degrees with ideal class groups of exponents $\leq 2$ for which the 2-ranks of the Galois group of $N$ over $\mathbb{Q}$ are equal to 2 .
\end{abstract}

\section{INTRODUCTION}

Chowla Cho proved that there are only finitely many imaginary quadratic fields with class groups of exponents $\leq 2$ : there are at most 66 such fields. (See $[\mathrm{BK}$, $\mathrm{We}$, and Lou90. See also $\mathrm{HB}$ for the finiteness of the imaginary quadratic fields with ideal class groups of exponents $\leq 6$.) This finiteness has been extended to imaginary abelian number fields of 2-power degrees with ideal class groups of exponents $\leq 2$ in $[\mathrm{HH}]$. Recently it was shown that under the Generalized Riemann Hypothesis (GRH) the exponents of the ideal class groups of the CM-number fields go to infinity with the absolute values of their discriminants in $\mathrm{AD}$ ] and [LO]. All nonquadratic imaginary cyclic number fields of 2-power degrees with ideal class groups of exponents $\leq 2$ are unconditionally determined in [Lou95]. In this paper we prove the following.

Theorem 1. Assume the Generalized Riemann Hypothesis. There are exactly $632(=483+149)$ imaginary abelian number fields $N$ of 2-power degrees with ideal class groups of exponents $\leq 2$ for which the 2 -ranks of the Galois group of $N$ over $\mathbb{Q}$ are equal to 2 . These fields are of degree $\leq 16$, of conductor $\leq 233905(=5 \cdot 7 \cdot 41 \cdot 163)$, and of class number $\leq 32$. All of these fields are listed in Tables 510.

We will usually write $\left(2^{n_{1}}, \ldots, 2^{n_{k}}\right)$ for $\left(\mathbb{Z} / 2^{n_{1}} \mathbb{Z}\right) \oplus \cdots \oplus\left(\mathbb{Z} / 2^{n_{k}} \mathbb{Z}\right)$ for brevity.

In the proof of Theorem 1 we have assumed the truth of GRH in the cases where the Galois group of $N$ over $\mathbb{Q}$ is isomorphic to one of the three groups $(2,2),(4,2)$, and $(8,2)$. Except for those three cases we do not use GRH. We now give a brief outline of our method. For a number field $k$ we let $C l(k), h_{k}$, and $h_{k}^{+}$be the class group, the class number, and the narrow class number of $k$, respectively. We denote by $\exp (C l(k))$ the exponent of $C l(k)$. For an extension $k_{1} / k_{2}$ of number fields we denote by $\mathfrak{D}\left(k_{1} / k_{2}\right)$ its different. Let $N$ be an imaginary abelian number field whose Galois group $G(N / \mathbb{Q})$ is isomorphic to $\left(2^{m}, 2^{l}\right)$ with $m \geq$ $l \geq 1$. Then $N=K K^{\prime}$, where $K$ and $K^{\prime}$ are the two imaginary subfields of $N$ with

Received by the editor November 15, 2009 and, in revised form, November 29, 2010.

2010 Mathematics Subject Classification. Primary 11R29, 11R20.

Key words and phrases. Class group, class numbers, relative class numbers.

This research was supported by Grant KRF-2008-313-C00008.

(C)2011 American Mathematical Society Reverts to public domain 28 years from publication 
$G(K / \mathbb{Q}) \simeq\left(2^{m}, 2^{l-1}\right)$ and $G\left(K^{\prime} / \mathbb{Q}\right) \simeq\left(2^{m}, 2^{l-1}\right)$ or $\left(2^{m-1}, 2^{l}\right)$. Our proof consists of three parts: (i) We will show that if $\exp (C l(N)) \leq 2$, then $\exp (C l(K)) \leq 2$ or $\exp \left(C l\left(K^{\prime}\right)\right) \leq 2$. (ii) We determine all fields $N$ with $\exp (C l(N)) \leq 2$ such that $G(N / \mathbb{Q})$ are isomorphic to $(4,2),(8,2)$, or $(4,4)$. (Note that the fields $N$ with $\exp (C l(N)) \leq 2$ and $G(N / \mathbb{Q}) \simeq(2,2)$ are already known in $[\mathrm{AK}$ : there are 483 such fields.) (iii) We show that if $G(N / \mathbb{Q})$ is not isomorphic to any of the four groups above, then $\exp (C l(N))>2$. In Section 2 we survey some known results for class groups of number fields. We study in Section 3 the group of characters associated to the imaginary abelian number fields $N$ with $G(N / \mathbb{Q}) \simeq\left(2^{m}, 2^{l}\right), m \geq$ $l \geq 1$. In Section 4 we prove that if $\exp (C l(N)) \leq 2$, then either $\exp (C l(K)) \leq 2$ or $\exp \left(C l\left(K^{\prime}\right)\right) \leq 2$. If $\mathfrak{D}(N / K) \neq(1)$, then $\exp (C l(K))$ divides $\exp (C l(N))$ by class field theory. Assume now that $N / K$ is unramified. Then $N / F$ is an unramified cyclic extension for every imaginary cyclic subfield $F$ of $K$ of degree $2^{m}$. We will find estimates of the relative class number $h_{F}^{-}$of an imaginary cyclic subfield $F$ of $N$ from above and below. Using genus theory, we will show that if $\exp (C l(N)) \leq 2$, then any primitive Dirichlet character $\chi_{F}$ associated with $F$ is of the form $\chi_{F}=\varphi_{p} \varphi_{q} \chi^{\prime}$, where $\varphi_{p}$ is a primitive Dirichlet character of $p$-power conductor for some prime $p$ and of order $2^{m}, \varphi_{q}$ is a primitive Dirichlet Character of $q$-power conductor for some prime $q(\neq p)$ and of order $2^{l}$ or $2^{l+1}$ with $l+1 \leq m$, and $\chi^{\prime}$ is the trivial character or a quadratic character of conductor prime to $p q$. The ambiguous class number formula yields an upper bound $U(m, l, \omega)$ for $h_{N}^{-}$ the relative class number of $N$ in terms of $m, l$, and $\omega$ the number of ramified primes. Moreover, $h_{N}^{-}$can be factored as $h_{N}^{-}=\frac{1}{2^{2^{l}}-1} \prod_{F} h_{F}^{-}$, where the product runs over the $2^{l}$ imaginary cyclic subfields $F$ of $K$ of degree $2^{m}$. We deduce that if $\exp (C l(N)) \leq 2$, then $N$ has at least one imaginary cyclic subfield $F$ so that $h_{F}^{-} \leq\left(2^{2^{l}-1} U(m, l, \omega)\right)^{\frac{1}{2^{l}}}$. Combining this with the lower bound $L\left(m, f_{F}\right)$ for $h_{F}^{-}$in Lou97, we get the inequality $L\left(m, f_{F}\right) \leq\left(2^{2^{l}-1} U(m, l, \omega)\right)^{\frac{1}{2^{l}}}$ involving $m, l$, and $f_{F}$ where $f_{F}$ is the conductor of $F$. See (3.4) and (3.5) below. This inequality gives an upper bound for $m(m \geq l)$. For each fixed $m$ under the upper bound we obtain an upper bound for $f_{F}$ for each given pair of $(m, l)$ using the inequality mentioned above. Then we find all possible conductors $f_{F}$ and all possible fields $N$. Computing $h_{F}^{-}$we verify that if $\mathfrak{D}(N / K)=\mathfrak{D}\left(N / K^{\prime}\right)=(1)$, then $\exp (C l(N))>2$. Assume now that $\mathfrak{D}\left(N / K^{\prime}\right)$ is not trivial. From class field theory, if $\exp (C l(N)) \leq 2$, then $\exp \left(C l\left(K^{\prime}\right)\right) \leq 2$. To begin with we treat the fields $N$ with $l=1$, i.e., $G(N / \mathbb{Q}) \simeq$ $\left(2^{m}, 2\right)$. In Section 5 , we will prove unconditionally that if $G(N / \mathbb{Q}) \simeq\left(2^{m}, 2\right)$ with $m \geq 4$, then $\exp (C l(N))>2$. Moreover, under the Generalized Riemann Hypothesis we determine all fields $N$ such that $G(N / \mathbb{Q}) \simeq\left(2^{m}, 2\right)$ with $m \leq 3$ and $\exp (C l(N)) \leq 2$. In Section [6 we will determine unconditionally all fields $N$ such that $G(N / \mathbb{Q}) \simeq(4,4)$ and $\exp (C l(N)) \leq 2$. In Section 7 we will verify unconditionally that if $G(N / \mathbb{Q}) \simeq(8,4)$, then $\exp (C l(N))>2$. From this we can prove by induction on $m$ that if $m \geq 3$, then $\exp (C l(N))>2$ for every imaginary abelian number field $N$ with $G(N / \mathbb{Q}) \simeq\left(2^{m}, 4\right)$. This yields that if $m \geq l \geq 3$, then $\exp (C l(N))>2$. So we conclude that if $G(N / \mathbb{Q}) \simeq\left(2^{m}, 2^{l}\right)$ with $m \geq l \geq 1$ and if $2^{m} \geq 16$ or $2^{l} \geq 8$, then $\exp (C l(N))>2$. Finally, we compile our computational results in Tables 510. We have computed $C l(N)$ by using KASH $([\mathrm{KT}])$ and GP $(\underline{\mathrm{P}})$.

We close this section by noticing that our method is not efficient for determining all imaginary abelian number fields $N$ of 2-power degrees with $\exp (C l(N)) \leq 2$. 
Because by our method we cannot find $r$ such that if the 2-rank of $G(N / \mathbb{Q})$ is greater than $r$, then $\exp (C l(N))>2$. It seems that if the 2-rank of $G(N / \mathbb{Q})$ is greater than 4 , then $\exp (C l(N))>2$. (See $[\mathrm{AK}$.)

\section{Preliminary Results and notations}

In this section we will survey some known results for class numbers and class groups of number fields that will be used in the sequel. We continue with the notations of Section 1. For an extension of number fields $k / k_{0}$ we let $\mathbb{N}_{k / k_{0}}: k \rightarrow k_{0}$ be the field norm of extension $k / k_{0}, \mathcal{N}_{k / k_{0}}: C l(k) \rightarrow C l\left(k_{0}\right)$ the ideal class group norm, $i_{k / k_{0}}: C l\left(k_{0}\right) \rightarrow C l(k)$ the map induced by extension of ideals, and let $t_{k / k_{0}}$ be the number of prime ideals in $k_{0}$ which are ramified in $k / k_{0}$. When an extension of number fields $k / k_{0}$ is unramified at all of the finite and infinite primes we say for brevity that $k / k_{0}$ is unramified.

Proposition 2. Suppose that the extension $k / k_{0}$ contains no unramified abelian extension $L$ with $L \neq k_{0}$. Then the norm map $\mathcal{N}_{k / k_{0}}$ is surjective. In particular, $h_{k_{0}}$ divides $h_{k}$ and $\exp \left(C l\left(k_{0}\right)\right)$ divides $\exp (C l(k))$.

Proof. See Theorem 5 in Appendix of Wa.

When $k$ is a CM-number field, we let $k^{+}$denote its maximal totally real subfield. According to Proposition $2, h_{k^{+}}$divides $h_{k}$. The quotient denoted by $h_{k}^{-}\left(=h_{k} / h_{k^{+}}\right)$ is called the relative class number of $k$. For a number field $k$ we let $O_{k}$ be its ring of integers, $O_{k}^{*}$ the group of units of $O_{k}$. For a quadratic extension $k / k_{0}$ of number fields let $A m\left(k / k_{0}\right)=\left\{c \in C l(k) \mid c=c^{\sigma}\right\}$ be its group of ambiguous ideal classes, where $\sigma$ denotes the nontrivial $k_{0}$-automorphism of $k$. For a finite abelian group $A$ we set $r_{2}(A)=\operatorname{dim}_{\mathbb{F}_{2}}\left(A / A^{2}\right)$ and call it the 2-rank of $A$.

Proposition 3. (1) Let $k / k_{0}$ be a quadratic extension of the number fields. Then

$$
\left|A m\left(k / k_{0}\right)\right|=\frac{h_{k_{0}} 2^{t^{\prime} k_{0}}-1}{\left[O_{k_{0}}^{*}: O_{k_{0}}^{*} \cap \mathbb{N}_{k / k_{0}}\left(k^{*}\right)\right]},
$$

where $t_{k / k_{0}}^{\prime}$ is the number of ramified primes including infinite primes in $k / k_{0}$. In particular, if $k$ is a CM-field, then

$$
\left|A m\left(k / k^{+}\right)\right|=h_{k^{+}} 2^{t_{k / k^{+}}{ }^{-1}}\left[O_{k^{+}}^{*} \cap \mathbb{N}_{k / k^{+}}\left(k^{*}\right): O_{k^{+}}^{*}\right] .
$$

(2) Let $k / k_{0}$ be a quadratic extension of the number fields and let $r_{2}=r_{2}(C l(k))$. Assume that $k / k_{0}$ is not unramified. Then the quotient $h_{k} / h_{k_{0}}$ divides

$$
\frac{h_{k}}{2^{r_{2}}}\left|A m\left(k / k_{0}\right)\right|
$$

In particular, if $k$ is a $C M$-field, then $h_{k}^{-}$divides

$$
\frac{h_{k}}{2^{r_{2}}}\left|A m\left(k / k^{+}\right)\right| \text {. }
$$

(3) If $k$ is a CM-field with class group of exponent $\leq 2$, then

$$
h_{k}^{-}=\frac{\left|A m\left(k / k^{+}\right)\right|}{\left|\operatorname{ker}\left(i_{k / k^{+}}\right)\right|}=\frac{h_{k^{+}} 2^{t_{k / k^{+}}-1}\left[O_{k^{+}}^{*} \cap \mathbb{N}_{k / k^{+}}\left(k^{*}\right): O_{k^{+}}^{*}\right]}{\left|\operatorname{ker}\left(i_{k / k^{+}}\right)\right|},
$$

where $i_{k / k^{+}}$is the natural map. Moreover, $\left|\operatorname{ker} i_{k / k^{+}}\right|=1$ or 2 , and if the absolute norm of $\mathfrak{D}\left(k / k^{+}\right)$has an odd prime divisor, then $\left|\operatorname{ker} i_{k / k^{+}}\right|=1$. 
(4) Let $k_{0}$ be a totally real number field with odd narrow class number $h_{k_{0}}^{+}$.

(i) For any real quadratic extension $k / k_{0}, h_{k}^{+}$is odd if and only if exactly one prime ideal of $k_{0}$ is ramified in $k$.

(ii) For any quadratic CM-extension $k / k_{0}$, we have $t_{k / k_{0}} \geq 1$ and

$$
r_{2}(C l(k))=t_{k / k_{0}}-1 .
$$

Proof. (1) See Che and [G].

(2) Let $\operatorname{ker}\left(\mathcal{N}_{k / k_{0}}\right)$ be the kernel of the norm map $\mathcal{N}_{k / k_{0}}$ and let $\mathfrak{B}=\{c \in$ $\left.C l(k) \mid c^{2}=1\right\}$. The injection

$$
\operatorname{ker}\left(\mathcal{N}_{k / k_{0}}\right) /\left(\operatorname{ker}\left(\mathcal{N}_{k / k_{0}}\right) \cap \mathfrak{B}\right) \hookrightarrow C l(k) / \mathfrak{B}
$$

yields that

$$
\frac{\left|\operatorname{ker}\left(\mathcal{N}_{k / k_{0}}\right)\right|}{\left|\operatorname{ker}\left(\mathcal{N}_{k / k_{0}}\right) \cap \mathfrak{B}\right|} \text { divides } \frac{h_{k}}{2^{r_{2}}} .
$$

Since $\left(\operatorname{ker}\left(\mathcal{N}_{k / k_{0}}\right) \cap \mathfrak{B}\right) \subset A m\left(k / k_{0}\right),\left|\operatorname{ker}\left(\mathcal{N}_{k / k_{0}}\right)\right|=h_{k} / h_{k_{0}}$ divides

$$
\frac{h_{k}}{2^{r_{2}}}\left|A m\left(k / k_{0}\right)\right|
$$

(3) The first statement follows from Sections 2 and 3 of $[\mathrm{E}]$. The second statement follows from Lemma 13.5 of $[\mathrm{CH}]$.

(4) For $(i)$ see (12.5) Corollary in $\mathrm{CH}$. For $($ ii) see Lemmas (13.6) and (13.7) in $\mathrm{CH}$.

For a Galois extension $k / k_{0}, G\left(k / k_{0}\right)$ denote its Galois group. For a number field $k$ we let $\operatorname{Hil}(k)$ be its Hilbert class field. If $k$ is an abelian extension of $\mathbb{Q}$ we denote by $\operatorname{Gen}(k), f_{k}$, and $X_{k}$ its genus field, its conductor, and the group of Dirichlet characters associated to $k$, respectively. For a primitive Dirichlet character $\chi$ we let $f(\chi)$ and $|\chi|$ be its conductor and its order, respectively. Throughout this paper a primitive Dirichlet character will be called a character for brevity. For a prime $p$, $\varphi_{p}$ denotes a primitive Dirichlet character for which $f\left(\varphi_{p}\right)$ is a power of $p$.

Proposition 4. Let $k / k_{0}$ be a cyclic unramified extension of number fields with $G\left(k / k_{0}\right) \simeq \mathbb{Z} / 2^{l} \mathbb{Z}$ and $l \geq 1$. Assume that $C l(k)$ has exponent $\leq 2$. Then we have the following.

(1) $\mathrm{Cl}\left(k_{0}\right)$ is isomorphic to

$$
\left(\mathbb{Z} / 2^{u} \mathbb{Z}\right) \oplus(\mathbb{Z} / 2 \mathbb{Z})^{v}
$$

where either $u=l$ or $u=l+1$, and $v \geq 0$.

(2) Suppose that $k / \mathbb{Q}$ is an abelian extension and $k_{0}$ is an imaginary cyclic number field of degree $2^{m}$ with $m \geq 2$. Let $\chi_{k_{0}}$ be any one of the generators of $X_{k_{0}}$. Let $f_{k_{0}}=\prod p^{e(p)}$ be the factorization of $f_{k_{0}}$ the conductor of $\chi_{k_{0}}$ and let

$$
\chi_{k_{0}}=\prod \varphi_{p}
$$

where $\varphi_{p}$ is a character of $p$ power conductor. Then:

(i) $f_{k_{0}}$ has one prime divisor $p$ with $\left|\varphi_{p}\right|=2^{m}$ and one another prime divisor $q$ such that $\left|\varphi_{q}\right|=2^{l}$ with $l \leq m$, or $\left|\varphi_{q}\right|=2^{l+1}$ with $l \leq m-1$.

(ii) If $f_{k_{0}}$ has more than two prime divisors $p$ and $q$, then $\left|\varphi_{r}\right|=2$ for prime $r$ with $r \mid f_{k_{0}}$ and $r \neq p, q$. 
(iii) If $p$ is odd, then $e(p)=1$ and $p \equiv 1\left(\bmod 2^{m}\right)$. If $p=2$, then $e(2)=m+2$. If $q$ is odd, then $e(q)=1$ and $q \equiv 1\left(\bmod 2^{l}\right)$ or $q \equiv 1$ $\left(\bmod 2^{l+1}\right)$. If $q=2$, then $e(q)=l+2$ or $l+3$.

Proof. (1) Since $k_{0} \subset k \subset H i l\left(k_{0}\right), G\left(k / k_{0}\right)$ is isomorphic to the factor group

$$
G\left(\operatorname{Hil}\left(k_{0}\right) / k_{0}\right) / G\left(\operatorname{Hil}\left(k_{0}\right) / k\right)
$$

which is isomorphic to $C l\left(k_{0}\right) / \mathcal{N}_{k / k_{0}}(C l(k))$ by the Takagi-Artin Theorem. In fact, according to Theorem 5.1 in Ch.VII of [CF], $G\left(k / k_{0}\right) \simeq$ $C_{k_{0}} / N_{k / k_{0}}\left(C_{k}\right)$, where $C_{k_{0}}\left(C_{k}\right.$ resp.) is the group of idèle classes of $k_{0}(k$ resp.), and $N_{k / k_{0}}: C_{k} \rightarrow C_{k_{0}}$ is the idèle class group norm. By using the natural map from the idèle group of $k_{0}$ to the group of fractional ideals of $k_{0}$ we get $C l\left(k_{0}\right) / \mathcal{N}_{k / k_{0}}(C l(k)) \simeq G\left(k / k_{0}\right)$. For details, see Ch.IV, $\S 8$ in [N]. The result follows immediately.

(2) From $\mathbb{Q} \subset k_{0} \subset k \subset \operatorname{Gen}\left(k_{0}\right) \subset \operatorname{Hil}(k)$ it follows that

$$
G\left(\operatorname{Gen}\left(k_{0}\right) / k_{0}\right) / G\left(G e n\left(k_{0}\right) / k\right) \simeq G\left(k / k_{0}\right) \simeq \mathbb{Z} / 2^{l} \mathbb{Z}
$$

and

$$
G\left(\operatorname{Gen}\left(k_{0}\right) / k\right) \simeq G(\operatorname{Hil}(k) / k) / G\left(\operatorname{Hil}(k) / G e n\left(k_{0}\right)\right) .
$$

As $C l(k) \simeq G(H i l(k) / k), G\left(G e n\left(k_{0}\right) / k\right)$ has exponent $\leq 2$ and

$$
G\left(\operatorname{Gen}\left(k_{0}\right) / k_{0}\right) \simeq\left(\mathbb{Z} / 2^{c} \mathbb{Z}\right) \oplus(\mathbb{Z} / 2 \mathbb{Z})^{d},
$$

where $c=l$ or $l+1$ and $d \geq 0$. Note that the group of characters associated to the genus field $\operatorname{Gen}\left(k_{0}\right)$ is generated by the $\varphi_{p}$ 's. Hence the character group $X_{k_{0}}$ is generated by the product of one character $\varphi_{p}$ of order $2^{m}$, one $\varphi_{q}$ with $p \neq q$ of order $2^{l}$ or $2^{l+1}$, and the other one(s) $\varphi_{r}$ of order 2 if $f_{k_{0}}$ has more than two prime divisors. This completes the proof.

Proposition 5. Let $F$ be an imaginary cyclic number field of degree $2 n=2^{m} \geq 4$ and let $d_{F}$ be the absolute value of the discriminant of $F$. Then we have

$$
h_{F}^{-} \geq \frac{2 \varepsilon_{F}}{\pi e \log d_{F}} \frac{f_{F}^{n / 2}}{\left(\pi \log f_{F^{+}}\right)^{n-1}}
$$

where $\varepsilon_{F}=\frac{2}{5} \exp \left(-\frac{2 \pi n}{d_{F}^{1 / 2 n}}\right)$ or $1-\frac{2 \pi n e^{1 / n}}{d_{F}^{1 / 2 n}}$.

Proof. See Remark 6 in [Lou97] and [R].

\section{The FIELDS $N$ with $G(N / \mathbb{Q}) \simeq\left(2^{m}, 2^{l}\right)$}

We consider the imaginary abelian number fields $N$ of which Galois group $G(N / \mathbb{Q})$ is isomorphic to $\left(2^{m}, 2^{l}\right)$ with $m \geq l \geq 1$ and $m \geq 2$. Let $\chi$ and $\psi$ be two generators of the character group $X_{N}$ with $|\chi|=2^{m}$ and $|\psi|=2^{l}$. We may assume $\chi(-1)=-1$ changing $\chi$ to $\chi \psi$ if necessary. The three subgroups of index 2 of $X_{N}$ are $\left\langle\chi, \psi^{2}\right\rangle,\left\langle\chi^{2}, \psi\right\rangle$, and $\left\langle\chi \psi, \psi^{2}\right\rangle$. We let $K$ be the imaginary subfield associated with the group $\left\langle\chi, \psi^{2}\right\rangle$ and let $K^{\prime}$ be the imaginary subfield associated with the group $\left\langle\chi \psi, \psi^{2}\right\rangle$ or $\left\langle\chi^{2}, \psi\right\rangle$ according as $\psi(-1)=1$ or -1 . We have $G\left(N^{+} / \mathbb{Q}\right)$ $\simeq\left(2^{m-1}, 2^{l}\right)$ or $\left(2^{m}, 2^{l-1}\right)$ according as $\psi(-1)=1$ or -1 .

Let $F$ be the imaginary cyclic subfield of degree $2^{m}$ over $\mathbb{Q}$ which is associated with the character group $\langle\chi\rangle$. 
Proposition 6. Let $N, K, K^{\prime}, \chi$ and $\psi$ be as above.

(1) If $\psi(-1)=-1$, then $\mathfrak{D}\left(N / K^{\prime}\right) \neq(1)$.

(2) Assume that $\exp (C l(N)) \leq 2$ and $\mathfrak{D}(N / K)=(1)$.

(a) If $\mathfrak{D}\left(N / K^{\prime}\right)=(1)$, then $l \leq m-1$ and $\chi$ can be written as

$$
\chi=\varphi_{p} \varphi_{q} \varphi_{f} \text { and } X_{N}=\left\langle\chi, \varphi_{q}^{2}\right\rangle,
$$

where $\left|\varphi_{p}\right|=2^{m},\left|\varphi_{q}\right|=2^{l+1}$, and $\varphi_{f}$ is a character of conductor $f$ with $\left|\varphi_{f}\right|=1$ or 2 and $(p q, f)=1$. Moreover, $\mathbb{Q}(\sqrt{p}, \sqrt{q}) \subset N^{+}$.

(b) If $\mathfrak{D}\left(N / K^{\prime}\right) \neq(1)$, then $\chi$ can be written as

$$
\chi=\varphi_{p} \varphi_{q} \varphi_{f} \text { and } X_{N}=\left\langle\chi, \varphi_{q} \varphi_{f^{\prime}}\right\rangle,
$$

where $\left|\varphi_{p}\right|=2^{m},\left|\varphi_{q}\right|=2^{l}, \varphi_{f}$ is a character of conductor $f$ with $\left|\varphi_{f}\right|=1$ or 2 and $(p q, f)=1, f^{\prime}$ is a divisor of $f$, and $\varphi_{f^{\prime}}$ is a character of conductor $f^{\prime}$ with $\left|\varphi_{f^{\prime}}\right|=1$ or 2 . If $m \geq l \geq 2$, then $\mathbb{Q}(\sqrt{p}, \sqrt{q}) \subset N^{+}$.

Proof. Let $f(\chi)=\prod p_{i}^{a_{i}}$. We write $\chi=\prod \varphi_{p_{i}}$ with $\left|\varphi_{p_{1}}\right|=2^{m}$. For a normal extension $k / k_{0}$ of number fields and a prime ideal $\mathfrak{p}$ of $k_{0}$ we let $e_{k / k_{0}}(\mathfrak{p})$ be the ramification index of $\mathfrak{p}$ in $k$.

(1) Let $F^{\prime}$ be the imaginary cyclic subfield associated with $\langle\psi\rangle$. If $m>l$, then the prime ideal(s) lying above $p_{1}$ must be ramified in $N / F^{\prime}$ and so in $N / K^{\prime}$ because $K^{\prime} \supset F^{\prime}, e_{N / \mathbb{Q}}\left(p_{1}\right) \geq 2^{m}>2^{l}$, and $N / F^{\prime}$ is a cyclic extension. We now assume that $m=l$. Let $S_{F}=\left\{p_{i} \mid p_{i}\right.$ is totally ramified in $\left.F\right\}$ and $S_{F^{\prime}}=\left\{q \mid q\right.$ is a prime that is totally ramified in $\left.F^{\prime}\right\}$. We have $S_{F} \neq S_{F^{\prime}}$. Otherwise $\mathbb{Q}(\sqrt{d}) \subset F \cap F^{\prime}$ and so $[N: \mathbb{Q}]<2^{m+l}$, where $d=\prod_{p \in S_{F}} p$. For $p_{j} \in S_{F} \backslash S_{F^{\prime}}$ we have $e_{N / \mathbb{Q}}\left(p_{j}\right) \geq e_{K / \mathbb{Q}}\left(p_{j}\right) \geq 2^{m}, e_{F^{\prime} / \mathbb{Q}}\left(p_{j}\right) \leq 2^{m-1}$, and thus the prime ideal(s) lying above $p_{j}$ must be ramified in $N / K^{\prime}$.

(2) By Proposition 4 point (2), we have $\chi=\prod \varphi_{p_{i}}$, where $\left|\varphi_{p_{1}}\right|=2^{m},\left|\varphi_{p_{2}}\right|=$ $2^{l+1}$ or $2^{l}$, and $\left|\varphi_{p_{i}}\right|=1$ or 2 for $i \neq 1,2$. For a prime $p$ we let $I_{p}$ be the inertia field of $p$ in the extension $N / \mathbb{Q}$.

(a) Suppose $\mathfrak{D}\left(N / K^{\prime}\right)=(1)$. We claim that $\left|\varphi_{p_{2}}\right|=2^{l+1}$. Since $I_{p_{1}} \subset$ $N^{+} \subset N, I_{p_{1}} \not \subset K$, and $I_{p_{1}} \not \subset K^{\prime}$. So $N / I_{p_{1}}$ is a cyclic extension of degree $2^{m}$ and $I_{p_{1}} / \mathbb{Q}$ is a cyclic extension of degree $2^{l}$. Note that every prime ideal lying above $p_{i}$ is ramified in $N / N^{+}$because $\mathfrak{D}(N / K)=\mathfrak{D}\left(N / K^{\prime}\right)=(1)$. Hence the only ramified prime in $I_{p_{1}} / \mathbb{Q}$ is $p_{2}$. Moreover, $p_{2}$ is totally ramified in $I_{p_{1}} / \mathbb{Q}$ because $I_{p_{1}} / \mathbb{Q}$ is cyclic, and the prime ideal(s) lying above $p_{2}$ is(are) also ramified in $N / N^{+}$. Hence $e_{N / \mathbb{Q}}\left(p_{2}\right)=\left|\varphi_{p_{2}}\right|=2^{l+1}$. As $F \subset N \subset G e n(F)$ and $X_{G e n(F)}$ is generated by $\varphi_{p_{i}} \mathrm{~s}, X_{I_{p_{1}}}$ is a cyclic group of order $2^{l}$ generated by $\varphi_{p_{2}}^{2}$. Thus, $X_{N}=\left\langle\chi, \varphi_{p_{2}}^{2}\right\rangle$.

(b) To begin with we will show that if $\left|\varphi_{p_{2}}\right|=2^{l+1}$, then $\mathfrak{D}\left(N / K^{\prime}\right)=(1)$. Let $G_{2^{l+1}}$ be the subfield of $\operatorname{Gen}(F)$ associated with $\left\langle\chi, \varphi_{p_{2}}\right\rangle$. Then $G\left(G_{2^{l+1}} / F\right)$ is a cyclic group of order $2^{l+1}$. We claim that $N \subset G_{2^{l+1}}$. Because $G_{2^{l+1}} N \subset \operatorname{Hil}(N)$ and $G\left(G_{2^{l+1}} N / N\right) \simeq G\left(G_{2^{l+1}} / G_{2^{l+1}} \cap N\right)$, this group is an elementary 2-group and $F \subset\left(G_{2^{l+1}} \cap N\right) \subset G_{2^{l+1}}$, so $\left[G_{2^{l+1}}: G_{2^{l+1}} \cap N\right]=2,\left[G_{2^{l+1}} \cap N: F\right]=2^{l}=[N: F]$, and $G_{2^{l+1}} \cap N=N$. Thus, $N \subset G_{2^{l+1}}$. Moreover, $G\left(G_{2^{l+1}} / K^{+}\right) \simeq(4,2)$, $G\left(G_{2^{l+1}} / N^{+}\right) \simeq(2,2)$, and $G\left(G_{2^{l+1}} / K\right) \simeq G\left(G_{2^{l+1}} / K^{\prime}\right) \simeq(\mathbb{Z} / 4 \mathbb{Z})$. 
As $K^{\prime} \subset N \subset G_{2^{l+1}}$ and $G_{2^{l+1}} / N$ is unramified, $G_{2^{l+1}} / K^{\prime}$ is unramified and so is $N / K^{\prime}$ as required. Hence $\mathfrak{D}\left(N / K^{\prime}\right) \neq(1)$ implies $\left|\varphi_{p_{2}}\right|=2^{l}$. In a similar way as in (a) we have that $I_{p_{1}} / \mathbb{Q}$ is cyclic and $X_{I_{p_{1}}}$ is generated by the character $\varphi_{p_{2}} \Pi^{\prime} \varphi_{p_{j}}$, where $\Pi^{\prime}$ runs through a possible subset of $p_{j}$ 's in $\chi=\prod \varphi_{p_{i}}$ with $\left|\varphi_{p_{j}}\right| \leq 2$.

The next proposition gives an upper bound for $h_{N}^{-}$.

Let $(\dot{\bar{p}})$ denote the Kronecker symbol.

Proposition 7. Let $N$ be an imaginary abelian number field with $G(N / \mathbb{Q}) \simeq$ $\left(2^{m}, 2^{l}\right)$ and $G\left(N^{+} / \mathbb{Q}\right) \simeq\left(2^{m-1}, 2^{l}\right)$. Assume that $X_{N}=\langle\chi, \psi\rangle$, where $\chi=$ $\varphi_{p} \varphi_{q} \varphi_{f}, \chi(-1)=-1,\left|\varphi_{p}\right|=2^{m},\left|\varphi_{q}\right|=2^{l+1}$ with $m \geq l+1, \varphi_{f}$ is a character of conductor $f$ with $\left|\varphi_{f}\right|=1$ or 2 and $(p q, f)=1$, and $\psi=\varphi_{q}^{2}$. Set $2 a=2^{m}$ and $2 b=2^{l}$. Suppose that $\exp \left(C l\left(N^{+}\right)\right) \leq 2$. Then we have the following.

(1) $h_{N}^{-}$divides $\frac{h_{N}}{2^{r}}\left|A m\left(N / N^{+}\right)\right|$, where $r=r_{2}(C l(N))$ is the 2-rank of $C l(N)$.

(2) $h_{N^{+}}$divides $2^{(a-1)(2 b-1)}$. Moreover, if $\left(\frac{p}{q}\right)=\left(\frac{q}{p}\right)=-1$, then $h_{N^{+}}=h_{N^{+}}^{+}=$ 1 and $\left|A m\left(N / N^{+}\right)\right|=2^{t_{N / N^{+}}-1}$.

(3) $\left[O_{N^{+}}^{*} \cap \mathbb{N}_{N / N^{+}}\left(N^{*}\right):\left(O_{N^{+}}^{*}\right)^{2}\right]$ divides $2^{a(2 b-1)}$.

$$
t_{N / N^{+}} \leq a+2 b+2 a b \omega(f)
$$

where $\omega(f)$ is the number of prime divisors of $f$. Moreover, if $\left(\frac{p}{q}\right)=\left(\frac{q}{p}\right)=$ -1 , then $t_{N / N^{+}} \leq 2+2 a b \omega(f)$.

(5) $h_{N}^{-}$divides $\left(\frac{h_{N}}{2^{r}}\right) 2^{2 a b(\omega(f)+2)-a}$.

Moreover, if $\left(\frac{p}{q}\right)=\left(\frac{q}{p}\right)=-1$, then $h_{N}^{-}$divides $\left(\frac{h_{N}}{2^{r}}\right) 2^{2 a b \omega(f)+1}$.

$$
h_{N}^{-}=\frac{1}{2^{2 b-1}} \prod_{F} h_{F}^{-}
$$

where $F$ runs over the imaginary cyclic subfields of degree $2^{m}$ of $N$.

(7) There exist at least one imaginary cyclic subfield $F$ of $N$ of degree $2 a=2^{m}$ such that

$$
h_{F}^{-} \leq 2^{a(\omega(f)+2-1 /(2 b))}\left(2^{2 b-1} \frac{h_{N}}{2^{r}}\right)^{1 /(2 b)} .
$$

In addition, if $\exp (C l(N)) \leq 2$, then (3.1) can be replaced by

$$
h_{F}^{-} \leq 2^{a(\omega(f)+2-1 /(2 b))} .
$$

If $\left(\frac{p}{q}\right)=\left(\frac{q}{p}\right)=-1$, then (3.1) can be replaced by

$$
h_{F}^{-} \leq 2^{a \omega(f)+1}\left(\frac{h_{N}}{2^{r}}\right)^{1 /(2 b)} .
$$

Moreover, if $\exp (C l(N)) \leq 2$ and $\left(\frac{p}{q}\right)=\left(\frac{q}{p}\right)=-1$, then (3.1) can be replaced by

$$
h_{F}^{-} \leq 2^{a \omega(f)+1} .
$$

For such a field $F$ we have

$$
\frac{e}{\varepsilon_{F}}\left(\frac{\log d_{F}}{\log f_{F^{+}}}\right)\left(2^{2 b-1} \frac{h_{N}}{2^{r}}\right)^{1 /(2 b)} \geq 2\left(\frac{\sqrt{f_{F}}}{2^{\omega(f)+2-1 /(2 b)} \pi \log f_{F^{+}}}\right)^{a} .
$$


In addition, if $\left(\frac{p}{q}\right)=\left(\frac{q}{p}\right)=-1$, then

$$
\frac{e}{\varepsilon_{F}}\left(\frac{\log d_{F}}{\log f_{F^{+}}}\right)\left(\frac{h_{N}}{2^{r}}\right)^{1 /(2 b)} \geq\left(\frac{\sqrt{f_{F}}}{2^{\omega(f)} \pi \log f_{F^{+}}}\right)^{a} .
$$

(8) $h_{\mathbb{Q}(\sqrt{p})}=h_{\mathbb{Q}(\sqrt{q})}=1, h_{\mathbb{Q}(\sqrt{p}, \sqrt{q})}=1$ or 2 , and $h_{\mathbb{Q}(\sqrt{p q})}=2 h_{\mathbb{Q}(\sqrt{p}, \sqrt{q})}$. Moreover, if $\left(\frac{p}{q}\right)=\left(\frac{q}{p}\right)=-1$, then $h_{\mathbb{Q}(\sqrt{p}, \sqrt{q})}=1$.

(9) Suppose that $\exp (C l(N)) \leq 2$. Let $K$ and $K^{\prime}$ be two imaginary subfields of $N$ associated with $\left\langle\chi, \psi^{2}\right\rangle$, respectively, $\left\langle\chi \psi, \psi^{2}\right\rangle$. If $\left(\frac{p}{q}\right)=\left(\frac{q}{p}\right)=-1$, then $h_{N}^{-}=2^{t_{N / N^{+}}-1}, h_{K}=h_{K^{\prime}}=2^{t_{K / K^{+}}}$, and $\operatorname{Hil}(K)=\operatorname{Hil}\left(K^{\prime}\right)$.

Proof. (1) It follows from Proposition 3 point (2).

(2) We have $X_{N^{+}}=\left\langle\varphi_{p}^{2}, \varphi_{q}^{2}\right\rangle$. Let $T$ be the cyclic subfield of $N^{+}$associated with $\left\langle\varphi_{p}^{2}\right\rangle$. By Proposition 3 point (4), $h_{T}=h_{T}^{+}=1$ because $p$ is totally ramified in $T / \mathbb{Q}$ and the prime ideal lying above $p$ is the unique ramified prime ideal. Consider the sequence of subfields $T_{i}$ of $N^{+}$,

$$
T_{0}=T \subset T_{1} \subset \cdots \subset T_{l}=N^{+}
$$

with $\left[T_{i}: T_{i-1}\right]=2$ for $i=1, \ldots, l$ where $2^{l}=2 b$. The prime ideal(s) lying above $q$ is (are) the only ramified ideal(s) in $T_{i} / T_{i-1}$. Since $\exp \left(C l\left(N^{+}\right)\right) \leq$ $2, \exp \left(C l\left(T_{i}\right)\right) \leq 2$ for each $i$ by Proposition 2. According to Proposition 3 points (1) and (2),

$$
\frac{h_{T_{i}}}{h_{T_{i-1}}} \text { divides }\left|A m\left(T_{i} / T_{i-1}\right)\right|
$$

and $\left|A m\left(T_{i} / T_{i-1}\right)\right|$ divides $h_{T_{i-1}} 2^{t_{T_{i} / T_{i-1}-1}}$. Hence,

$$
h_{T_{i}} \text { divides } h_{T_{i-1}}^{2} 2^{t_{T_{i} / T_{i-1}}-1}
$$

and so

$$
h_{N^{+}} \text {divides } 2^{\alpha} \text {, }
$$

where

$$
\begin{aligned}
\alpha & =2^{l-1}\left(t_{T_{1} / T_{0}}-1\right)+2^{l-2}\left(t_{T_{2} / T_{1}}-1\right)+\cdots+\left(t_{T_{l} / T_{l-1}}-1\right) \\
& \leq(a-1)\left(2^{l}-1\right)=(a-1)(2 b-1)
\end{aligned}
$$

since $t_{T_{i} / T_{i-1}} \leq a$ for each $i$. If $\left(\frac{p}{q}\right)=\left(\frac{q}{p}\right)=-1$, then $h_{T_{i}}=h_{T_{i}}^{+}=1$ for $i=1, \ldots, l$, by Proposition 3 point (4). In particular, $h_{N^{+}}=h_{N^{+}}^{+}=$ 1 and so $O_{N^{+}}^{*} \cap \mathbb{N}_{N / N^{+}}\left(N^{*}\right)=O_{N^{+}}{ }^{2}$. (See Section $12[\mathrm{CH}]$.) Hence $\left|A m\left(N / N^{+}\right)\right|=2^{t}{ }_{N / N^{+}}^{-1}$ by Proposition 3 point (1).

(3) Let $O_{T}^{*+}$ be the set of totally positive units of $T$. Set $E=O_{N^{+}}^{*} \cap$ $\mathbb{N}_{N / N^{+}}\left(N^{*}\right)$. Since every element in $E \subset \mathbb{N}_{N / N^{+}}\left(N^{*}\right)$ is totally positive and $h_{T}=h_{T}^{+}=1, O_{T}^{*+}=O_{T}^{* 2}=E \cap O_{T}^{*}$. From the injection

$$
O_{T}^{*} / O_{T}^{* 2} \hookrightarrow O_{N^{+}}^{*} / E
$$

it follows that $\left|O_{T}^{*} / O_{T}^{* 2}\right|=2^{a}$ divides $\left|O_{N^{+}}^{*} / E\right|$. Moreover,

$$
O_{N^{+}}^{*} / E \simeq\left(O_{N^{+}}^{*} / O_{N^{+}}^{*}{ }^{2}\right) /\left(E / O_{N^{+}}^{*}{ }^{2}\right)
$$

and so

$$
\left|O_{N^{+}}^{*} / E\right|=\frac{2^{2 a b}}{\left|E / O_{N^{+}}^{*}{ }^{2}\right|} .
$$


Thus

$$
\left|E / O_{N^{+}}^{*}\right| \text { divides } 2^{a(2 b-1)}
$$

(4) It is clear.

(5) The first statement follows from (1), (2), (3), (4) and Proposition 3 point (1). Suppose that $\left(\frac{p}{q}\right)=\left(\frac{q}{p}\right)=-1$. Then, $h_{N^{+}}=h_{N^{+}}^{+}=1$ from (2). So, the second statement follows from (1)-(4).

(6) We have

$$
h_{N}^{-}=Q_{N} w_{N} \prod_{\substack{1 \leq i \leq 2 a, i o d d \\ 1 \leq j \leq 2 b}} \frac{1}{2} L\left(0, \chi^{i} \psi^{j}\right),
$$

where $Q_{N}$ is the Hasse unit index of $N$ and $w_{N}$ is the number of roots of unity in $N$. (See $\S \S 20-27$ in $\mathrm{Ha}$, Ch. 4 in $\mathrm{Wa}$, and Lou01.) Here, $Q_{N}=1$ because the prime ideals lying above $p$ and $q$ are ramified in $N / N^{+}$. There are precisely $2 b$ imaginary cyclic subfields $F_{j}$ with which the character group associated are $\left\langle\chi \psi^{j}\right\rangle$ for $1 \leq j \leq 2 b$. Furthermore,

$$
h_{F_{j}}^{-}=Q_{F_{j}} w_{F_{j}} \prod_{\substack{i \text { odd } \\ 1 \leq i \leq 2 a}} \frac{1}{2} L\left(0, \chi^{i} \psi^{i j}\right) .
$$

Note that $Q_{F_{j}}=1, w_{F_{j}}=2$, and $w_{N}=2$. Then the result follows.

(7) It follows from (5), (6), and Proposition 5 .

(8) Since $p$ and $q$ are 2 or odd primes congruent to $1 \bmod 4, \mathbb{Q}(\sqrt{p}) \subset N^{+}$, and $\mathbb{Q}(\sqrt{q}) \subset N^{+}, h_{\mathbb{Q}(\sqrt{p})}=h_{\mathbb{Q}(\sqrt{q})}=1$. Let $B=\mathbb{Q}(\sqrt{p}, \sqrt{q})$. We claim that $\exp (C l(B)) \leq 2$. Note that $X_{B}=\left\langle\varphi_{p}^{a}, \varphi_{q}^{2 b}\right\rangle$. Let $A$ be the subfield of $N$ associated to the character group $\left\langle\varphi_{p}^{a}, \varphi_{q}^{2}\right\rangle$. Then the extension $N^{+} / A$ $(A / B$ resp.) is a cyclic extension in which any prime lying above $p$ ( $q$ resp.) is totally ramified. By Proposition 2, $C l(A)$ and $C l(B)$ both have exponent $\leq 2$. From $\exp (C l(B)) \leq 2$ it follows that $C l(B)=A m(B / \mathbb{Q}(\sqrt{p}))$. By Proposition 3 point $(1), h_{B}=1$ or 2 . Moreover, if $\left(\frac{p}{q}\right)=\left(\frac{q}{p}\right)=-1$, then $h_{B}=1$. Hence $h_{\mathbb{Q}(\sqrt{p q})}=2$ if $h_{B}=1, \operatorname{Hil}(B)=\operatorname{Hil}(\mathbb{Q}(\sqrt{p q}))$ and $h_{\mathbb{Q}(\sqrt{p q})}=4$ if $h_{B}=2$.

(9) By (2), $h_{N^{+}}=h_{N^{+}}^{+}=1$. From Proposition 3 point (4) it follows that $h_{N}=2^{t_{N / N^{+}}{ }^{-1}}$. Note that $t_{N / N^{+}} \leq 2\left(t_{K / K^{+}}-2\right)+2$ and so $h_{N} \leq$ $2^{2 t_{K / K^{+}}-3}$. Since $\mathfrak{D}\left(N^{+} / K^{+}\right)=(1), 2 \mid h_{K^{+}}$. Moreover, $h_{K^{+}} \mid 2 h_{N^{+}}$by [Mas, Corollaries 2.2 and 2.3]. So $h_{K^{+}}=2$. By Proposition 3 point (1),

$$
h_{K} \geq\left|A m\left(K / K^{+}\right)\right| \geq 2^{t_{K / K^{+}}} \text {. }
$$

Similarly, $h_{K^{\prime}} \geq 2^{t_{K / K^{+}}}$. As $h_{N}^{-}=\frac{1}{2} h_{K}^{-} h_{K^{\prime}}^{-}$and

$$
h_{N}=h_{N^{-}}^{-} h_{N^{+}}=\frac{h_{K} h_{K^{\prime}} h_{N^{+}}}{2 h_{K^{+}}^{2}}=\frac{h_{K} h_{K^{\prime}}}{8},
$$

$h_{K}=\left|A m\left(K / K^{+}\right)\right|=2^{t_{K / K^{+}}}=\left|A m\left(K^{\prime} / K^{+}\right)\right|=h_{K^{\prime}}$. Hence both $\operatorname{Hil}(K) / K^{+}$and $\operatorname{Hil}\left(K^{\prime}\right) / K^{+}$are abelian, so $\operatorname{Hil}(K)=\operatorname{Hil}\left(K^{\prime}\right)$.

4. The FIELDS $N=K K^{\prime}$ WITH $\mathfrak{D}(N / K)=\mathfrak{D}\left(N / K^{\prime}\right)=(1)$

The notation here is that introduced in the previous sections. In this section, using the upper bounds for $h_{N}^{-}$and $h_{F}^{-}$in Proposition 7 we will show the following.

Proposition 8. If $\mathfrak{D}(N / K)=\mathfrak{D}\left(N / K^{\prime}\right)=(1)$, then $\exp (C l(N))>2$. 
Proof. Contrary to the conclusion, we suppose that $\exp (C l(N)) \leq 2$. By Proposition 6 point (2),

$$
X_{N}=\langle\chi, \psi\rangle
$$

with $\chi=\varphi_{p} \varphi_{q} \varphi_{f}, \psi=\varphi_{q}^{2},\left|\varphi_{p}\right|=2 a,\left|\varphi_{q}\right|=4 b, a \geq 2 b, \chi(-1)=-1$, and $\varphi_{f}$ is a character of conductor $f$ with $\left|\varphi_{f}\right|=1$ or 2 and $(p q, f)=1$. Set $f=r_{1} r_{2} \cdots r_{g}$ or 1 , where $r_{i}$ 's are 4,8 , or odd primes. According to Proposition 7 there is at least one imaginary cyclic subfield $F$ of $N$ of degree $2 a$ over $\mathbb{Q}$ for which the inequality (3.2) or (3.3) holds. We will find all imaginary cyclic subfields $F$ that satisfy the inequalities (3.2) or (3.3) if $\left(\frac{p}{q}\right)=\left(\frac{q}{p}\right)=-1$. Our computations were divided into six cases:

Case 1: $\left(\frac{p}{q}\right)=\left(\frac{q}{p}\right)=1, p=2$ and $q$ is odd.

Case 2: $\left(\frac{p}{q}\right)=\left(\frac{q}{p}\right)=1, p$ is odd and $q=2$.

Case 3: $\left(\frac{p}{q}\right)=\left(\frac{q}{p}\right)=1$, both $p$ and $q$ are odd.

Case 4: $\left(\frac{p}{q}\right)=\left(\frac{q}{p}\right)=-1, p=2$ and $q$ is odd.

Case 5: $\left(\frac{p}{q}\right)=\left(\frac{q}{p}\right)=-1, p$ is odd and $q=2$.

Case 6: $\left(\frac{p}{q}\right)=\left(\frac{q}{p}\right)=-1$, both $p$ and $q$ are odd.

Following Louboutin's approach in Lou95 we proceed in each of the six cases as follows.

(i) We get an upper bound for $a$ by using (3.4) or (3.5).

(ii) For each of fixed $a$ and $b$ which are lower than the upper bound found in (i) we get an upper bound for $f_{F}$ the conductor of $F$.

(iii) We find all possible conductors $f_{F}$ of $F$ which satisfy (3.4) or (3.5) and compute $h_{F}^{-}$.

(iv) For each of those characters we verify whether for all the $2 b$ fields $F$ with given conductor $f_{F}, h_{F}^{-}$are powers of 2 as well as there is at least one field $F$ that satisfies (3.2) or (3.3). In Cases 1-3 there is no such conductor. In Cases 4-6 there are in all two such conductors. But all of these two conductors are ruled out by Proposition 7 point (9). From this we conclude $\exp (C l(N))>2$.

To illustrate our method we give the details of our computations for Case 1 . For the remaining cases we summarize our computational results at the end of the proof.

Case $1\left(p=2\right.$ and $q$ is odd with $\left.\left(\frac{2}{q}\right)=1\right)$. Since $f_{F}=8 a q f, f_{F^{+}}=4 a q$, and $d_{F}=2^{4 a-1} a^{2 a} q^{2 a-a /(2 b)} f^{a}$, (3.4) yields

$$
\begin{gathered}
C_{F}(a, b, q, f) \cdot a \cdot\left(\frac{\log \left(2^{4-1 / a} a^{2} q^{2-1 /(2 b)} f\right)}{\log (4 a q)}\right) \\
\quad \geq 2\left(\frac{\sqrt{8 a q f}}{2^{\omega(f)+2-1 /(2 b)} \pi \log (4 a q)}\right)^{a}
\end{gathered}
$$

where $C_{F}(a, b, q, f)=e / \varepsilon_{F}$. From (4.6) we will get upper bounds for $a, q$, and $f$. Our computations consist of five parts: (1)-(5).

(1) We take

$$
\varepsilon_{F}=1-\frac{2 \pi a e^{1 / a}}{d_{F}^{1 /(2 a)}} .
$$


Since

$$
C_{F}(a, b, q, f)=e\left(1-\frac{\pi(\sqrt{2} e)^{1 / a}}{2 q^{1-1 /(4 b)} f^{1 / 2}}\right)^{-1}
$$

is decreasing as a function of $a, b, q$ and $f$, respectively, we have

$$
C_{F}(a, b, q, f) \leq C_{F}(2,1,17,1) \leq e\left(1-\frac{\pi e^{1 / 2}}{(34)^{3 / 4}}\right)^{-1} \leq 4.31 .
$$

(2) For a nonnegative integer $j$ we let $f_{j}=p_{0} p_{1} \cdots p_{j}$, where $p_{0}=1$ and $\left(p_{i}\right)_{i \geq 1}$ is the increasing sequence of odd primes. Note that the function

$$
x \mapsto \frac{x^{a / 2}}{C_{F}(a, b, q, x) \log \left(2^{4-1 / a} a^{2} q^{2-1 /(2 b)} x\right)}
$$

is increasing on $[1, \infty)$ and $f \geq f_{\omega}$ with $\omega=\omega(f) \geq 0$. Hence, (4.6) yields

$$
C_{F}\left(a, b, q, f_{\omega}\right) \cdot a \cdot\left(\frac{\log \left(2^{4-1 / a} a^{2} q^{2-1 /(2 b)} f_{\omega}\right)}{\log (4 a q)}\right) \geq 2\left(\frac{\sqrt{8 a q f_{\omega}}}{2^{\omega+2-1 /(2 b)} \pi \log (4 a q)}\right)^{a} .
$$

(3) We let

$$
g(\omega)=\frac{f_{\omega}^{a / 2}}{2^{a \omega} \log \left(2^{4-1 / a} a^{2} q^{2-1 /(2 b)} f_{\omega}\right)} .
$$

Then $g(0)>g(1)$ and $g(\omega) \leq g(\omega+1)$ for $\omega \geq 1$. So,

$$
g(\omega) \geq g(1)
$$

for all $\omega \geq 0$ and hence (4.8) yields

$C_{F}(a, b, q, 1) \cdot a \cdot\left(\frac{\log \left(2^{4-1 / a} a^{2} q^{2-1 /(2 b)} 3\right)}{\log (4 a q)}\right) \geq 2\left(\frac{\sqrt{24 a q}}{2^{3-1 /(2 b)} \pi \log (4 a q)}\right)^{a}$.

(4) On the left-hand side of (4.9) the function

$$
x \mapsto C_{F}(a, b, x, 1)\left(\frac{\log \left(2^{4-1 / a} a^{2} x^{2-1 /(2 b)} 3\right)}{\log (4 a x)}\right)
$$

is decreasing on $[17, \infty)$ because

$$
\log \left(2^{1 / b-1 / a} 3 a^{1 /(2 b)}\right) \geq \log 3>0 .
$$

Moreover, on the right-hand side of (4.9) the function

$$
x \mapsto \frac{\sqrt{x}}{\log (4 a x)}
$$

is increasing on $[17, \infty)$. Hence, it follows from (4.9) that

$C_{F}(a, b, 17,1) \cdot a \cdot\left(\frac{\log \left(2^{4-1 / a} a^{2} 17^{2-1 /(2 b)} 3\right)}{\log (68 b)}\right) \geq 2\left(\frac{\sqrt{408 a}}{2^{3-1 /(2 b)} \pi \log (68 a)}\right)^{a}$

(5) By (4.7) and 4.10

$4.31 \cdot a \cdot\left(\frac{\log \left(2^{4-1 / a} a^{2} 17^{2-1 / a} 3\right)}{\log (68 a)}\right) \geq 2\left(\frac{\sqrt{408 a}}{2^{3-1 / a} \pi \log (68 a)}\right)^{a}$,

which yields $2 \leq a \leq 2^{7}$. Let us fix $a$ and $b$ with $b<a$. We can get an upper bound for $q$ by using (4.9). Since $\mathbb{Q}(\sqrt{q}, \sqrt{2 q}) \subset N^{+}, q$ has to satisfy 
$h_{\mathbb{Q}(\sqrt{q})}=1$ and $h_{\mathbb{Q}(\sqrt{2 q})}=2$ or 4 . For each such $q$ we get an upper bound for $\omega$. Indeed, (4.8) yields

$$
\left(\frac{(\log 4 a q)^{a-1}}{2 a^{a / 2-1}}\right)\left(\frac{2^{2-1 /(2 b)} \pi}{\sqrt{8 q}}\right)^{a} \geq \frac{g(\omega)}{C_{F}\left(a, b, q, f_{\omega}\right)} .
$$

Note that

$$
\omega \mapsto \frac{g(\omega)}{C_{F}\left(a, b, q, f_{\omega}\right)}
$$

tends to infinity as $\omega$ tends to infinity. Finally, for each $q$ and $\omega$ we can get an upper bound for $f$ by using the inequality (4.6). Then we can make a list of all possible conductors. There are 3668 possible conductors in all. Our computational results are summarized in Table 1 .

TABLE 1. $p=2$ and $q \equiv 1 \bmod 4 b$

\begin{tabular}{|c|c|c|c|c|c|}
\hline$a$ & $b$ & $q \leq$ & $\omega \leq$ & $f \leq$ & $\sharp\{(q, \omega, f)\}$ \\
\hline 2 & 1 & 3049 & 6 & 285285 & 2111 \\
\hline 4 & 1 & 937 & 5 & 15015 & 391 \\
\cline { 2 - 6 } & 2 & 1433 & 5 & 26565 & 702 \\
\hline 8 & 1 & 281 & 4 & 1365 & 84 \\
\cline { 2 - 6 } & 2 & 457 & 4 & 2415 & 152 \\
\cline { 2 - 6 } & 4 & 433 & 4 & 3003 & 101 \\
\hline 16 & 1 & 97 & 3 & 165 & 21 \\
\cline { 2 - 6 } & 2 & 137 & 3 & 231 & 38 \\
\cline { 2 - 6 } & 4 & 193 & 4 & 1155 & 30 \\
\cline { 2 - 6 } & 8 & 193 & 2 & 21 & 6 \\
\hline 32 & 1 & 17 & 2 & 21 & 5 \\
\cline { 2 - 6 } & 2 & 41 & 3 & 105 & 10 \\
\cline { 2 - 6 } & 4 & 17 & 3 & 105 & 9 \\
\cline { 2 - 6 } & $8 \sim 16$ & None & None & None & 0 \\
\hline 64 & 1 & 17 & 1 & 3 & 1 \\
\cline { 2 - 6 } & 2 & 17 & 2 & 15 & 3 \\
\cline { 2 - 6 } & 4 & 17 & 2 & 15 & 4 \\
\cline { 2 - 6 } & $8 \sim 32$ & None & None & None & 0 \\
\hline 128 & $1 \sim 64$ & None & None & None & 0 \\
\hline
\end{tabular}

For each of those quintuples $(a, b, q, \omega, f)$ we compute $h_{F}^{-}$for all the $2 b$ imaginary cyclic number fields $F$ of conductor $f_{F}=8 a q f$ and degree $2 a$, and verify that $h_{F}^{-}$ is not a power of 2 for at least one such $F$. We conclude that in this case there is no field $N$ with $\exp (C l(N)) \leq 2$.

Case 2 ( $p$ is odd and $q=2$ with $\left(\frac{p}{2}\right)=1$ ). In this case we have $4 \leq a \leq 2^{5}$ and there are 1347 quintuples of $(a, b, p, \omega, f)$. For each of those quintuple $(a, b, p, \omega, f)$ computing $h_{F}^{-}$for all the $2 b$ imaginary cyclic number fields $F$ of conductor $f_{F}=$ $16 b p f$ and degree $2 a$ we verify that $h_{F}^{-}$is not a power of 2 for at least one such $F$. Hence in this case there is no field $N$ with $\exp (C l(N)) \leq 2$.

Case 3 (both $p$ and $q$ are odd with $\left(\frac{p}{q}\right)=1$ ). In this case we have $2 \leq a \leq 2^{7}$ and there are 23598 sextuples of $(a, b, p, q, \omega, f)$. For each of those 23598 sextuples 
$(a, b, p, q, \omega, f)$ computing $h_{F}^{-}$for all the $2 b$ imaginary cyclic number fields $F$ of conductor $f_{F}=p q f$ and degree $2 a$ we verify that there are exactly two sextuples such that $h_{F}^{-}$'s are powers of 2 for all $2 b$ fields $F$ of conductor $p q f$ : those two sextuples are given in Table 2 But for these two sextuples $h_{F}^{-}$'s do not satisfy the inequality (3.2).

TABLE 2 .

\begin{tabular}{|c|c|c|c|}
\hline$N r$. & $(a, b, p, q, \omega, f)$ & $h_{F}^{-\prime} \mathrm{s}$ & $2^{a(\omega+2-1 /(2 b))}$ \\
\hline 1 & $(2,1,41,61,1,5)$ & $2^{8}, 2^{10}$ & $2^{5}$ \\
\hline 2 & $(2,1,5,101,1,59)$ & $2^{9}, 2^{13}$ & $2^{5}$ \\
\hline
\end{tabular}

Hence, in this case there is no field $N$ with $\exp (C l(N)) \leq 2$.

Case 4. $p=2$ and $q$ is odd with $\left(\frac{2}{q}\right)=-1$ ] In this case we have $2 \leq a \leq 2^{4}$ and there are 628 quintuples of $(a, b, q, \omega, f)$. For each of those 628 quintuples $(a, b, q, \omega, f)$ computing $h_{F}^{-}$for all the $2 b$ imaginary cyclic number fields of conductor $f_{F}=8 a q f$ and degree $2 a$ we verify that there is only one quintuple $(a, b, q, \omega, f)$ such that $h_{F}^{-}$'s are powers of 2 for all the $2 b$ fields $F:(a, b, q, \omega, f)=(2,1,5,1,3)$ with $\left(h_{F_{1}}^{-}, h_{F_{2}}^{-}\right)=(8,16)$, where $F_{1}$ and $F_{2}$ are two cyclic fields of conductor 240 and degree 4. However, $N=F_{1} F_{2}$ has $\exp (C l(N))>2$. Otherwise, $F_{1}$ and $F_{2}$ would have $h_{F_{1}}=h_{F_{2}}$ by Proposition 7 point (9). Hence, in this case there is no field $N$ with $\exp (C l(N)) \leq 2$.

Case 5 ( $p$ is odd and $q=2$ with $\left(\frac{p}{2}\right)=-1$ ). Exchanging $p$ with $q$ if $a=2 b$ we are in the case 4 . So we may assume that $a \geq 4 b \geq 4$. But, in this case we have $\left(\frac{p}{2}\right)=1$ since 8 divides $p-1$. Hence, we do not need to consider this case.

Case 6 (both $p$ and $q$ are odd with $\left(\frac{p}{q}\right)=-1$ ). In this case we have $2 \leq a \leq 2^{4}$ and there are 3128 sextuples of $(a, b, p, q, \omega, f)$ in all. For all of those sextuples $(a, b, p, q, \omega, f)$ we compute $h_{F}^{-}$and verify that there are exactly four sextuples such that $h_{F}^{-1}$ S are power of 2 for all the $2 b$ fields $F$ of conductor $p q f$ and degree $2 a$. Among them there is only one sextuple $(a, b, p, q, \omega, f)$ for which there is a field $F$ satisfying (3.3): $(a, b, p, q, \omega, f)=(2,1,5,13,1,4)$. For this sextuple we let $F_{1}$ and $F_{2}$ be two imaginary cyclic fields of conductor 260 and degree 4 and let $N=F_{1} F_{2}$. We have $\left(h_{F_{1}}^{-}, h_{F_{2}}^{-}\right)=(8,16)$. From the fact that $h_{F_{1}} \neq h_{F_{2}}$, it follows that $\exp (C l(N))>2$ by Proposition 7 point(9). Our computational results are given in Table 3 ,

TABLE 3 .

\begin{tabular}{|c|c|c|c|}
\hline$N r$. & $(a, b, p, q, \omega, f)$ & $h_{F}^{-\prime} \mathrm{s}$ & $2^{a \omega+1}$ \\
\hline 1 & $(2,1,5,13,1,4)$ & 8,16 & 8 \\
\hline 2 & $(2,1,5,17,2,21)$ & 64,64 & 32 \\
\hline 3 & $(2,1,13,37,2,20)$ & 256,256 & 32 \\
\hline 4 & $(2,1,5,53,3,276)$ & 4096,4096 & 128 \\
\hline
\end{tabular}


According to our computational result we conclude that there is no field $N$ with $\exp (C l(N)) \leq 2$.

This completes the proof of Proposition 8 .

\section{The Fields $N$ with $G(N / \mathbb{Q}) \simeq\left(2^{m}, 2\right)$}

In this section we assume that $N$ is an imaginary abelian number field with $G(N / \mathbb{Q}) \simeq\left(2^{m}, 2\right)$ and $m \geq 1$. The aim of this section is to determine all fields $N$ with $\exp (C l(N)) \leq 2$ and $m \geq 2$. Note that all such fields with $m=1$ are known under the Generalized Riemann Hypothesis in $\mathrm{AK}$. Let notation be the same as Sections 3 and 4 . The field $N$ has two imaginary subfields $K$ and $K^{\prime}$ such that $N=$ $K K^{\prime},[N: K]=\left[N: K^{\prime}\right]=2$ and $G(K / \mathbb{Q}) \simeq \mathbb{Z} / 2^{m} \mathbb{Z}$. According to Proposition 8 if $\exp (C l(N)) \leq 2$, then $\mathfrak{D}(N / K)) \neq(1)$ or $\mathfrak{D}\left(N / K^{\prime}\right) \neq(1)$. Changing $\chi$ to $\chi \psi$ if necessary we may assume that $\mathfrak{D}\left(N / K^{\prime}\right) \neq(1)$ and so $\exp \left(C l\left(K^{\prime}\right)\right) \leq 2$ by Proposition 2. First, we determine all fields $N=K K^{\prime}$ with $\exp (C l(K))>2$ and $\exp (C l(N)) \leq 2$. Second, we look for all fields $N=K K^{\prime}$ with $\exp (C l(K)) \leq 2$ and $\exp (C l(N)) \leq 2$.

Proposition 9. Let $2 a=2^{m}$ with $m \geq 2$. Assume that $\exp (C l(N)) \leq 2$. Suppose that $N$ has an imaginary cyclic subfield $K$ with $\exp (C l(K))>2$. Let $\chi$ be any one of the odd character with $X_{K}=\langle\chi\rangle$. Then we have the following.

(1) The character $\chi$ is of the form

$$
\chi=\varphi_{p} \varphi_{f} \text { with } \chi(-1)=-1,
$$

where $\left|\varphi_{p}\right|=2 a, f\left(\varphi_{p}\right)= \begin{cases}p & \text { if } p \text { is odd, } \\ 8 a & \text { if } p=2,\end{cases}$

and $\varphi_{f}$ is a character of conductor $f$ with $\left|\varphi_{f}\right|=2$ and $p \nmid f$.

(2) $h_{K^{+}}^{+}=h_{K^{+}}=1, h_{K}^{-}=2^{t_{K / K^{+}}}$, and $C l(K) \simeq(\mathbb{Z} / 4 \mathbb{Z}) \oplus(\mathbb{Z} / 2 \mathbb{Z})^{t_{K / K^{+}}-2}$.

(3)

$$
\frac{e}{\varepsilon_{K}} \cdot a \cdot\left(\frac{\log \left(d_{K}^{1 / a}\right)}{\log \left(f_{K^{+}}\right)}\right) \geq\left(\frac{\sqrt{f_{K}}}{2^{\omega(f)} \pi \log \left(f_{K^{+}}\right)}\right)^{a},
$$

where $\left(f_{K}, f_{K^{+}}, d_{K}\right)= \begin{cases}\left(8 a f, 4 a, 2^{4 a-1} a^{2 a} f^{a}\right) & \text { if } p=2, \\ \left(p f, p, p^{2 a-1} f^{a}\right) & \text { if } p>2 .\end{cases}$

(4) $2 \leq a \leq 64$ and $f \leq 3.3 \times 10^{6}$.

(5) $G(N / \mathbb{Q}) \simeq(4,2), G\left(N^{+} / \mathbb{Q}\right) \simeq(2,2)$, and $N$ is one of the four fields listed in Table 5 .

Proof. (1) It follows from Proposition 6 point (2).

(2) The first statement follows from Proposition 3 point (4). The second statement follows from Proposition 4 point (1) and Proposition 3 point (4).

(3) It follows from point (2) and Proposition 5

(4) and (5) Using similar arguments as in Section 4 we get the results.

Corollary 10. Let $N$ be an imaginary abelian number field with $G(N / \mathbb{Q}) \simeq\left(2^{m}, 2\right)$, $m \geq 4$. Then we have unconditionally $\exp (C l(N))>2$.

Proof. Suppose $\exp (C l(N)) \leq 2$. By Proposition 9 point (5), if $K$ is an imaginary cyclic subfield of $N$ with $[N: K]=2$ then $\exp (C l(K)) \leq 2$. According to Lou95] $[K: \mathbb{Q}]=2^{m}=16$ and $X_{K}=\left\langle\chi_{17}\right\rangle$, where $\chi_{17}$ is an odd character of conductor 
17 and of order 16 . Then $X_{N}$ is of the form $X_{N}=\left\langle\chi_{17}, \psi\right\rangle$, where $\psi$ is a quadratic character. Furthermore, $\psi$ must be odd. Otherwise, there are two imaginary cyclic subfields $K$ of $N$ with $[N: K]=2$ and $\exp (C l(K)) \leq 2$, which contradicts Theorem 11 in Lou95. We claim that $\psi$ must be $\chi_{4}$ the quadratic character of conductor 4 . Let $N_{16}$ and $k_{8}$ be subfields of $N$ associated with $\left\langle\chi_{17}^{2}, \psi\right\rangle$ and $\left\langle\chi_{17}^{2} \psi\right\rangle$, respectively. As 17 divides the absolute norm of $\mathfrak{D}\left(N / N_{16}\right), \exp \left(C l\left(N_{16}\right)\right) \leq 2$. By Proposition 9 point $(5), \exp \left(C l\left(k_{8}\right)\right) \leq 2$; for $G\left(N_{16} / \mathbb{Q}\right) \simeq(8,2)$. Let $N_{8}$ and $k_{4}$ be the subfield of $N$ associated with $\left\langle\chi_{17}^{4}, \psi\right\rangle$ and $\left\langle\chi_{17}^{4} \psi\right\rangle$, respectively. By a similar argument as above $\exp \left(C l\left(N_{8}\right)\right) \leq 2$ and $\exp \left(C l\left(k_{4}\right)\right) \leq 2$. According to Theorems 12 and 13 in Lou95 the fact that $\exp \left(C l\left(k_{8}\right)\right) \leq 2$ and $\exp \left(C l\left(k_{4}\right)\right) \leq 2$ implies $\psi=\chi_{4}$. However, we verify that the field $N_{8}$ associated with $\left\langle\chi_{17}^{4}, \chi_{4}\right\rangle$ has $C l\left(N_{8}\right) \simeq \mathbb{Z} / 4 \mathbb{Z}$. This contradiction shows that $\exp (C l(N))>2$.

Proposition 11. Let $N$ be an imaginary abelian number fields with $G(N / \mathbb{Q}) \simeq$ $\left(2^{m}, 2\right)$ and $m \geq 2$. Let $K$ and $K^{\prime}$ be two imaginary subfields of $N$ with $[N$ : $K]=\left[N: K^{\prime}\right]=2$ and $G(K / \mathbb{Q}) \simeq \mathbb{Z} / 2^{m} \mathbb{Z}$. Suppose that $\exp (C l(N)) \leq 2$, $\exp (C l(K)) \leq 2$, and $\exp \left(C l\left(K^{\prime}\right)\right) \leq 2$. Then we have the following.

(1) $m \leq 3$.

(2) If $G(N / \mathbb{Q}) \simeq(4,2)$ and $G\left(N^{+} / \mathbb{Q}\right) \simeq(2,2)$, then there are 38 such fields.

(3) If the Generalized Riemann Hypothesis is true and if $G(N / \mathbb{Q}) \simeq(4,2)$ and $G\left(N^{+} / \mathbb{Q}\right) \simeq \mathbb{Z} / 4 \mathbb{Z}$, then there are 100 such fields.

(4) If $G(N / \mathbb{Q}) \simeq(8,2)$ and $G\left(N^{+} / \mathbb{Q}\right) \simeq(4,2)$, then there is exactly one such field $N$ with $X_{N}=\left\langle\chi_{17}^{2} \chi_{4}, \chi_{4} \chi_{3}\right\rangle$, where $\chi_{3}$ is the quadratic odd character of conductor 3 .

(5) If the Generalized Riemann Hypothesis is true and if $G(N / \mathbb{Q}) \simeq(8,2)$ and $G\left(N^{+} / \mathbb{Q}\right) \simeq \mathbb{Z} / 8 \mathbb{Z}$, then there are 3 such fields.

All of those $142=(38+100+1+3)$ fields $N$ are compiled in Tables 6 . 9 .

Proof. (1) It follows from Corollary 10.

(2) and (4) By Lou95 there are 94 pairs of two imaginary cyclic fields $\left(K, K^{\prime}\right)$ with $\exp (C l(K)) \leq 2, \exp \left(C l\left(K^{\prime}\right)\right) \leq 2$, and $K^{+}=\left(K^{\prime}\right)^{+}$. For those pairs we compute the ideal class groups of the composita $N=K K^{\prime}$ and obtain the result. Similar arguments are used for (4).

(3) and (5) By Lou95 and AK there are 169 pairs $\left(K, K^{\prime}\right)$ of two imaginary fields such that $G(K / \mathbb{Q}) \simeq \mathbb{Z} / 4 \mathbb{Z}, G\left(K^{\prime} / \mathbb{Q}\right) \simeq(2,2), \exp (C l(K)) \leq 2$, $\exp \left(C l\left(K^{\prime}\right)\right) \leq 2$, and $K^{+}=\left(K^{\prime}\right)^{+}$. Note that in [AK] we determine all such fields $K^{\prime}$ under the Generalized Riemann Hypothesis. For those pairs we compute the ideal class groups of the composita $N=K K^{\prime}$ and obtain the result. (5) can be shown similarly.

\section{The FIELDS $N$ With $G(N / \mathbb{Q}) \simeq(4,4)$}

The aim of this section is to prove

Proposition 12. There are three fields $N$ with $G(N / \mathbb{Q}) \simeq(4,4)$ for which $C l(N)$ have exponents $\leq 2$. Those fields are listed in Table 10 .

Proof. Let $\chi$ ( $\psi$ resp.) be an odd (even resp.) character of order 4 such that $X_{N}=\langle\chi, \psi\rangle$. Let $K\left(K^{\prime}\right.$ resp.) be the subfield associated with the group $\left\langle\chi, \psi^{2}\right\rangle$ $\left(\left\langle\chi \psi, \psi^{2}\right\rangle\right.$ resp.). By Proposition 8 we may assume that $\mathfrak{D}\left(N / K^{\prime}\right) \neq(1)$ and so $C l\left(K^{\prime}\right)$ has exponent $\leq 2$. We consider two cases separately. 
(1) Assume that $\exp (C l(K)) \leq 2$. By using Propositions 9 and 11 there are four pairs of $\left(K, K^{\prime}\right)$ such that $G(K / \mathbb{Q}) \simeq(4,2)$ with $G\left(K^{+} / \mathbb{Q}\right) \simeq(2,2)$, $G\left(K^{\prime} / \mathbb{Q}\right) \simeq(4,2)$ with $G\left(K^{\prime+} / \mathbb{Q}\right) \simeq(2,2), G(N / \mathbb{Q}) \simeq(4,4)$ with $N=$ $K K^{\prime}, K^{+}=\left(K^{\prime}\right)^{+}, \exp (C l(K)) \leq 2$, and $\exp \left(C l\left(K^{\prime}\right)\right) \leq 2$. We verify that among those four pairs there are three pairs for which $N=K K^{\prime}$ has $\exp (C l(N)) \leq 2$. Those fields are listed in Table 10 .

(2) Assume that $\exp (C l(K))>2$. According to Propositions 9and11there are $42=(4+38)$ fields $K^{\prime}$ such that $G\left(K^{\prime} / \mathbb{Q}\right) \simeq(4,2)$ with $G\left(K^{\prime+} / \mathbb{Q}\right) \simeq(2,2)$ and $\exp \left(C l\left(K^{\prime}\right)\right) \leq 2$. Each of these fields $K^{\prime}$ contains the real subfield $\mathbb{Q}(\sqrt{p}, \sqrt{q})$, where $(p, q) \in S=\{(2,5),(2,13),(2,17),(5,13),(5,17),(5,41)$, $(5,61),(13,17),(13,29)\}$. If $\exp (C l(N)) \leq 2$ and $\exp (C l(K))>2$, then we have the following.

(i) $\mathfrak{D}(N / K)=(1)$ by Proposition 2, hence $\mathfrak{D}(N / F)=(1)$ for any imaginary cyclic quartic subfields $F$ of $K$.

(ii) $C l(K) \simeq(\mathbb{Z} / 4 \mathbb{Z}) \oplus(\mathbb{Z} / 2 \mathbb{Z})^{v}$ for some integer $v \geq 0$ by Proposition 4 point (1).

(iii) $C l(F) \simeq\left(\mathbb{Z} / 2^{2+\epsilon} \mathbb{Z}\right) \oplus(\mathbb{Z} / 2 \mathbb{Z})^{v_{1}}$ with $\epsilon=0$ or 1 and $v_{1} \leq v$ for any imaginary cyclic quartic subfields $F$ of $K$ by Proposition 4 point (1).

(iv) $\chi$ is of the form

$$
\chi=\varphi_{p} \varphi_{q} \varphi_{f},
$$

where $\left|\varphi_{p}\right|=\left|\varphi_{q}\right|=4$ with $(p, q) \in S$, and $\varphi_{f}$ is a character of conductor $f$ with $\left|\varphi_{f}\right| \leq 2$ and $(p q, f)=1$, by Proposition 6 point (2).

$(v)$ By Proposition $7, h_{K}^{-} \leq 2^{4 \omega(f)+7}$ and the number field $K$ has at least one imaginary cyclic quartic subfield $F$ with $h_{F}^{-} \leq 2^{2 \omega(f)+4}$ and

$$
\frac{e}{\varepsilon_{F}}\left(\frac{\log \left(d_{F}^{1 / 2}\right)}{\log \left(f_{F^{+}}\right)}\right) \geq\left(\frac{\sqrt{f_{F}}}{2^{\omega(f)+2} \pi \log \left(f_{F^{+}}\right)}\right)^{2},
$$

which yields $f_{F} \leq 8.7 \times 10^{6}$.

We verify that there are exactly three triplets $(p, q, f)$ such that $h_{F}^{-}$is a power of 2 for both of two imaginary cyclic quartic subfields $F$ of $K$ : $(2,5,3),(5,13,4)$, and $(5,17,21)$. But, each of those three triplets $(p, q, f)$ has at least one imaginary cyclic quartic subfield $F$ such that $C l(F)^{2}$ is not cyclic, which is contradictory to (iii). Our computational results are given in Table 4, the character $\chi_{4}$ ( the same as in the proof of Corollary 10 above), $\chi_{p}$ for each odd prime $p$, and $\psi_{2^{i}}$ for each integer $i \geq 3$ are defined at the beginning of Section 9.

TABLE 4.

\begin{tabular}{|c|c|c|c|c|c|}
\hline$N r$. & $(p, q, f)$ & $F_{1}$ & $C l\left(F_{1}\right)$ & $F_{2}$ & $C l\left(F_{2}\right)$ \\
\hline 1 & $(2,5,3)$ & $\left\langle\psi_{16} \chi_{4} \chi_{5} \chi_{3}\right\rangle$ & $(4,2,2)$ & $\left\langle\psi_{16} \chi_{4} \chi_{5}^{3} \chi_{3}\right\rangle$ & $(4,4,2)$ \\
\hline 2 & $(5,13,4)$ & $\left\langle\chi_{5} \chi_{13}^{3} \chi_{4}\right\rangle$ & $(4,2,2)$ & $\left\langle\chi_{5} \chi_{13}^{9} \chi_{4}\right\rangle$ & $(4,4,2)$ \\
\hline 3 & $(5,17,21)$ & $\left\langle\chi_{5} \chi_{17}^{4} \chi_{7}^{3} \chi_{3}\right\rangle$ & $(4,4,2,2,2)$ & $\left\langle\chi_{5} \chi_{17}^{12} \chi_{7}^{3} \chi_{3}\right\rangle$ & $(4,4,2,2,2)$ \\
\hline
\end{tabular}

We conclude that there is no field $N=K K^{\prime}$ with $\exp (C l(N)) \leq 2, \exp (C l(K))>$ 2 , and $\exp \left(C l\left(K^{\prime}\right)\right) \leq 2$. This completes the proof of Proposition 12 


\section{The FIELDS $N$ WITH $G(N / \mathbb{Q}) \simeq(8,4)$}

Proposition 13. If $N$ is an imaginary abelian number field with $G(N / \mathbb{Q}) \simeq(8,4)$, then $\exp (C l(N))>2$.

Proof. Let $\chi$ and $\psi$ be the two generators of $X_{N}$ with $X_{N}=\langle\chi, \psi\rangle,|\chi|=8$, $\chi(-1)=-1$, and $|\psi|=4$. Let $K$ be the subfield associated with $\left\langle\chi, \psi^{2}\right\rangle$ and let $K^{\prime}$ be the subfield associated with $\left\langle\chi \psi, \psi^{2}\right\rangle$ or $\left\langle\chi^{2}, \psi\right\rangle$ according as $\psi(-1)=1$ or -1 . Suppose $\exp (C l(N)) \leq 2$. We consider two cases separately.

(i) Assume $\psi(-1)=1$. Then $G\left(N^{+} / \mathbb{Q}\right) \simeq(4,4), G(K / \mathbb{Q}) \simeq G\left(K^{\prime} / \mathbb{Q}\right) \simeq$ $(8,2)$, and $G\left(K^{+} / \mathbb{Q}\right) \simeq G\left(K^{+} / \mathbb{Q}\right) \simeq(4,2)$. By Proposition $8, \mathfrak{D}(N / K) \neq$ (1) or $\mathfrak{D}\left(N / K^{\prime}\right) \neq(1)$. Say $\mathfrak{D}\left(N / K^{\prime}\right) \neq(1)$. So, $C l\left(K^{\prime}\right)$ has exponent $\leq 2$. Moreover, $K^{\prime}$ is associated with $\left\langle\chi_{17}^{2} \chi_{4}, \chi_{4} \chi_{3}\right\rangle, \exp (C l(K))>2$, and $\mathfrak{\mathfrak { D }}(N / K)=(1)$ by Proposition 11 point (4). By Proposition 6 point (2), $\chi$ is of the form $\chi=\varphi_{p} \varphi_{q} \varphi_{f}$ and $X_{N}=\left\langle\chi, \varphi_{q} \varphi_{f^{\prime}}\right\rangle$, where $\left|\varphi_{p}\right|=8,\left|\varphi_{q}\right|=4$, $\varphi_{f}$ is a character of conductor $f$ with $\left|\varphi_{f}\right|=1$ or 2 and $(p q, f)=1, f^{\prime}$ is a divisor of $f$, and $\varphi_{f^{\prime}}$ is a character of conductor $f^{\prime}$ with $\left|\varphi_{f}^{\prime}\right|=1$ or 2. Hence, the unique biquadratic bicyclic subfield $\mathbb{Q}(\sqrt{p}, \sqrt{q})$ of $N^{+}$is $\mathbb{Q}(\sqrt{17}, \sqrt{3})$. This is absurd; for $p \equiv 1(\bmod 8)$ and $q \equiv 1(\bmod 4)$.

(ii) Assume $\psi(-1)=-1$. Then $G\left(N^{+} / \mathbb{Q}\right) \simeq(8,2), G(K / \mathbb{Q}) \simeq(8,2)$ with $G\left(K^{+} / \mathbb{Q}\right) \simeq(4,2)$, and $G\left(K^{\prime} / \mathbb{Q}\right) \simeq(4,4)$ with $G\left(K^{\prime+} / \mathbb{Q}\right) \simeq(4,2)$. Then $\mathfrak{D}\left(N / K^{\prime}\right) \neq(1)$ and $\exp \left(C l\left(K^{\prime}\right)\right) \leq 2$. So, $K^{\prime}$ is one of three fields in Table 10. Note that there is no pair of $\left(K, K^{\prime}\right)$ such that $G(K / \mathbb{Q}) \simeq$ $(8,2)$ with $G\left(K^{+} / \mathbb{Q}\right) \simeq(4,2), \exp (C l(K)) \leq 2$, and $K^{+}=K^{\prime+}$. Hence, $\exp (C l(K))>2$ and $\mathfrak{D}(N / K)=(1)$. By Proposition 6 point (2), $\chi$ is of the form $\chi=\varphi_{p} \varphi_{q} \varphi_{f}$ and $X_{N}=\left\langle\chi, \varphi_{q} \varphi_{f^{\prime}}\right\rangle$, where $\left|\varphi_{p}\right|=8,\left|\varphi_{q}\right|=4, \varphi_{f}$ is a character of conductor $f$ with $\left|\varphi_{f}\right|=1$ or 2 and $(p q, f)=1, f^{\prime}$ is a divisor of $f$, and $\varphi_{f^{\prime}}$ is a character of conductor $f^{\prime}$ with $\left|\varphi_{f^{\prime}}\right|=1$ or 2 . Hence, $X_{K^{\prime}}$ is of the form $\left\langle\varphi_{p}^{2} \varphi_{q}^{2}, \varphi_{q}^{2}\right\rangle=\left\langle\varphi_{p}^{2}, \varphi_{q}^{2}\right\rangle$. This is absurd. Because according to Table 10, $X_{K^{\prime+}}=\left\langle\chi_{5} \psi_{16} \chi_{4}, \psi_{8}\right\rangle,\left\langle\chi_{5} \chi_{13}^{3}, \chi_{13}^{6}\right\rangle$ or $\left\langle\chi_{5} \psi_{16} \chi_{3}, \psi_{8}\right\rangle$.

This completes the proof.

\section{The FIELDS $N$ WITH $G(N / \mathbb{Q}) \simeq\left(2^{m}, 2^{l}\right)$ WITH $m \geq l$, AND $m \geq 4$ OR $l \geq 3$}

Proposition 14. If $N$ is an imaginary abelian number field with $G(N / \mathbb{Q}) \simeq$ $\left(2^{m}, 2^{l}\right)$ with $m \geq l$, and if $m \geq 4$ or $l \geq 3$, then $\exp (C l(N))>2$.

Proof. Let $\chi$ and $\psi$ be two generators of $X_{N}$ such that $|\chi|=2^{m}, \chi(-1)=-1$, $|\psi|=2^{l}$, and $X_{N}=\langle\chi, \psi\rangle$.

(i) By Corollary 10, if $m \geq 4$ and $l=1$, then $\exp (C l(N))>2$.

(ii) Assume $\psi(-1)=1$. We may assume that $m>l$ and hence $m \geq 4$ by the assumption. If $m=l$, then we change $\psi$ to $\chi \psi$ and will consider this in (iii) below. Assume $l \geq 2$. (For $l=1$ we have already considered in $(i)$ ). Let $K$ and $K^{\prime}$ be the subfields associated with $\left\langle\chi, \psi^{2}\right\rangle$ and $\left\langle\chi \psi, \psi^{2}\right\rangle$, respectively. Suppose that $C l(N)$ has exponent $\leq 2$. By Proposition $8, \mathfrak{D}(N / K) \neq(1)$ or $\mathfrak{D}\left(N / K^{\prime}\right) \neq(1)$. Say $\mathfrak{D}\left(N / K^{\prime}\right) \neq(1)$. So, $C l\left(K^{\prime}\right)$ has exponent $\leq 2$. In an iterative fashion we have that there is an imaginary subfield $F$ of $N$ such that $G(F / \mathbb{Q}) \simeq\left(2^{m}, 2\right)$ with $m \geq 4$ and $\exp (C l(F)) \leq 2$. This would contradict Corollary 10. Therefore, $\exp (C l(N))>2$. 
(iii) Assume $\psi(-1)=-1$. Note that $N$ has two imaginary subfield $K$ and $K^{\prime}$ such that $X_{K}=\left\langle\chi, \psi^{2}\right\rangle$ and $X_{K^{\prime}}=\left\langle\chi^{2}, \psi\right\rangle$. By Proposition 6 point(1), the extension $N / K^{\prime}$ is not unramified. (a) Claim that if $m \geq 4$ and $l=2$, then $\exp (C l(N))>2$. Suppose the contrary. By Proposition 2, $\exp \left(C l\left(K^{\prime}\right)\right)$ $\leq 2$. In an iterative fashion we would have an imaginary subfield $L$ with $G(L / \mathbb{Q}) \simeq(8,4)$ and $\exp (C l(L)) \leq 2$, which contradicts Proposition 13 , (b) Claim that if $l \geq 3$, then $\exp (C l(N))>2$. Suppose that $\exp (C l(N))$ $\leq 2$. By Proposition 6 point $(1), C l\left(K^{\prime}\right)$ has exponent $\leq 2$. In an iterative fashion we have that there is an imaginary subfield $M$ of $N$ such that $G(M / \mathbb{Q}) \simeq\left(2^{l}, 2^{l}\right)$ with $l \geq 3$ and $\exp (C l(M)) \leq 2$. We denote by $X_{M}=$ $\left\langle\chi_{0}, \psi_{0}\right\rangle$ with $\left|\chi_{0}\right|=2^{l},\left|\psi_{0}\right|=2^{l}, \chi_{0}(-1)=-1$, and $\psi_{0}(-1)=-1$. Let $M_{0}$ be the subfield associated with $\left\langle\chi_{0}, \psi_{0}^{2}\right\rangle$. Then $\exp \left(C l\left(M_{0}\right)\right) \leq 2$ by Proposition 6 point(1). By Proposition [13, $l \geq 4$. However, in $(i)$ above, we already showed that if $l \geq 4$, then $\exp \left(C l\left(M_{0}\right)\right)>2$. This contradiction implies $\exp (C l(N))>2$.

This completes the proof.

Theorem [1 follows from the proof of Theorem 1 in [AK], Propositions 9 , 11, 12, 13 and 14 .

\section{TABles}

For an odd prime $p, \chi_{p}$ denotes the odd character of conductor $p$ and of order $p-1$ with $\chi_{p}(g)=e^{2 \pi i /(p-1)}$, where $g$ is the smallest positive integer which generates the multiplicative group $(\mathbb{Z} / p \mathbb{Z})^{*}$. For prime $2, \chi_{4}$ denotes the odd quadratic character of conductor 4 and $\psi_{8}$ denotes the even quadratic character of conductor 8 .

When $i \geq 3$ we let $\psi_{2^{i}}$ be the even character of conductor $2^{i}$ and of order $2^{i-2}$ with $\psi_{2^{i}}^{2}=\psi_{2^{i-1}}$.

TABLE 5. $G(N / \mathbb{Q}) \simeq(4,2)$ with $G\left(N^{+} / \mathbb{Q}\right) \simeq(2,2), \exp (C l(N)) \leq$ $2, \exp (C l(K))>2$ and $\exp \left(C l\left(K^{\prime}\right)\right) \leq 2, K$ is associated with $\langle\chi\rangle$ and $K^{\prime}$ is associated with $\langle\chi \psi\rangle$.

\begin{tabular}{|c|c|c|c|c||c|c|c|c|c|}
\hline$N r$. & $X_{N}=\langle\chi, \psi\rangle$ & $h_{N}$ & $h_{K}$ & $h_{K^{\prime}}$ & $N r$. & $X_{N}=\langle\chi, \psi\rangle$ & $h_{N}$ & $h_{K}$ & $h_{K^{\prime}}$ \\
\hline 1 & $\left\langle\psi_{16} \chi_{4} \chi_{17}^{8}, \chi_{17}^{8}\right\rangle$ & 4 & 8 & 1 & 3 & $\left\langle\chi_{5} \chi_{61}^{30}, \chi_{61}^{30}\right\rangle$ & 4 & 8 & 1 \\
\hline 2 & $\left\langle\chi_{5} \chi_{41}^{20}, \chi_{41}^{20}\right\rangle$ & 4 & 8 & 1 & 4 & $\left\langle\chi_{13}^{3} \chi_{29}^{14}, \chi_{29}^{14}\right\rangle$ & 4 & 8 & 1 \\
\hline
\end{tabular}

TABLE 6. $G(N / \mathbb{Q}) \simeq(4,2)$ with $G\left(N^{+} / \mathbb{Q}\right) \simeq(2,2), \exp (C l(N)) \leq$ $2, \exp (C l(K)) \leq 2$, and $\exp \left(C l\left(K^{\prime}\right)\right) \leq 2, K$ is associated with $\langle\chi\rangle$ and $K^{\prime}$ is associated with $\langle\chi \psi\rangle$.

\begin{tabular}{|c|c|c|c|c||c|c|c|c|c|}
\hline$N r$. & $X_{N}=\langle\chi, \psi\rangle$ & $h_{N}$ & $h_{K}$ & $h_{K^{\prime}}$ & $N r$. & $X_{N}=\langle\chi, \psi\rangle$ & $h_{N}$ & $h_{K}$ & $h_{K^{\prime}}$ \\
\hline 1 & $\left\langle\chi_{5}, \psi_{8}\right\rangle$ & 1 & 1 & 2 & 20 & $\left\langle\chi_{13}^{3}, \chi_{17}^{8}\right\rangle$ & 2 & 1 & 4 \\
\hline 2 & $\left\langle\psi_{16} \chi_{4}, \chi_{3} \chi_{4}\right\rangle$ & 2 & 1 & 2 & 21 & $\left\langle\psi_{16} \chi_{3}, \chi_{3} \chi_{4} \chi_{5}^{2}\right\rangle$ & 4 & 2 & 2 \\
\hline 3 & $\left\langle\chi_{5}, \chi_{3} \chi_{4}\right\rangle$ & 2 & 1 & 4 & 22 & $\left\langle\chi_{13}^{3}, \chi_{3} \chi_{7}^{3}\right\rangle$ & 4 & 1 & 8 \\
\hline 4 & $\left\langle\chi_{13}^{3}, \chi_{5}^{2}\right\rangle$ & 1 & 1 & 2 & 23 & $\left\langle\chi_{5} \psi_{8}, \psi_{8} \chi_{4} \chi_{7}^{3}\right\rangle$ & 4 & 2 & 4 \\
\hline 5 & $\left\langle\chi_{5}, \chi_{13}^{6}\right\rangle$ & 1 & 1 & 2 & 24 & $\left\langle\chi_{5}, \chi_{3} \chi_{19}^{9}\right\rangle$ & 4 & 1 & 8 \\
\hline 6 & $\left\langle\psi_{16} \chi_{4}, \chi_{5}^{2}\right\rangle$ & 1 & 1 & 2 & 25 & $\left\langle\chi_{13}^{3} \psi_{8}, \psi_{8} \chi_{3} \chi_{4}\right\rangle$ & 8 & 2 & 8 \\
\hline
\end{tabular}




\begin{tabular}{|c|c|c|c|c||c|c|c|c|c|}
\hline 7 & $\left\langle\chi_{5}, \chi_{17}^{8}\right\rangle$ & 1 & 1 & 2 & 26 & $\left\langle\psi_{16} \chi_{3}, \chi_{3} \chi_{7}^{3}\right\rangle$ & 4 & 2 & 4 \\
\hline 8 & $\left\langle\chi_{13}^{3}, \psi_{8}\right\rangle$ & 1 & 1 & 2 & 27 & $\left\langle\chi_{5} \chi_{3} \chi_{4}, \chi_{4} \chi_{7}^{3}\right\rangle$ & 8 & 4 & 4 \\
\hline 9 & $\left\langle\chi_{5}, \chi_{3} \chi_{7}^{3}\right\rangle$ & 2 & 1 & 4 & 28 & $\left\langle\chi_{5} \chi_{3} \chi_{7}^{3}, \chi_{3} \chi_{4}\right\rangle$ & 8 & 4 & 4 \\
\hline 10 & $\left\langle\psi_{16} \chi_{4}, \chi_{4} \chi_{7}^{3}\right\rangle$ & 4 & 1 & 4 & 29 & $\left\langle\chi_{5} \chi_{3} \chi_{4}, \chi_{3} \chi_{7}^{3}\right\rangle$ & 8 & 4 & 4 \\
\hline 11 & $\left\langle\chi_{5} \chi_{3} \chi_{4}, \psi_{8}\right\rangle$ & 8 & 4 & 4 & 30 & $\left\langle\chi_{5} \psi_{8}, \psi_{8} \chi_{4} \chi_{11}^{5}\right\rangle$ & 8 & 2 & 8 \\
\hline 12 & $\left\langle\chi_{5}, \chi_{3} \chi_{4} \psi_{8}\right\rangle$ & 2 & 1 & 4 & 31 & $\left\langle\chi_{17}^{4} \chi_{7}^{3}, \chi_{4} \chi_{7}^{3}\right\rangle$ & 4 & 2 & 4 \\
\hline 13 & $\left\langle\chi_{5} \psi_{8}, \chi_{3} \chi_{4}\right\rangle$ & 4 & 2 & 4 & 32 & $\left\langle\psi_{16} \chi_{4} \chi_{5}^{2}, \chi_{4} \chi_{5}^{2} \chi_{7}^{3}\right\rangle$ & 8 & 2 & 4 \\
\hline 14 & $\left\langle\chi_{5} \psi_{8}, \chi_{3} \chi_{4} \psi_{8}\right\rangle$ & 4 & 2 & 4 & 33 & $\left\langle\chi_{5} \chi_{3} \chi_{7}^{3}, \chi_{7}^{3} \chi_{4} \psi_{8}\right\rangle$ & 8 & 4 & 4 \\
\hline 15 & $\left\langle\chi_{17}^{4} \chi_{4}, \psi_{8}\right\rangle$ & 8 & 4 & 4 & 34 & $\left\langle\chi_{17}^{4} \chi_{7}^{3}, \chi_{7}^{3} \chi_{4} \psi_{8}\right\rangle$ & 4 & 2 & 4 \\
\hline 16 & $\left\langle\chi_{5}, \chi_{7}^{3} \chi_{4}\right\rangle$ & 2 & 1 & 4 & 35 & $\left\langle\chi_{13}^{3} \chi_{5}^{2}, \chi_{5}^{2} \chi_{17}^{8}\right\rangle$ & 8 & 2 & 4 \\
\hline 17 & $\left\langle\chi_{13}^{3}, \chi_{3} \chi_{4}\right\rangle$ & 4 & 1 & 8 & 36 & $\left\langle\chi_{5} \chi_{3} \chi_{4}, \chi_{4} \chi_{19}^{9}\right\rangle$ & 16 & 4 & 8 \\
\hline 18 & $\left\langle\chi_{5}, \chi_{3} \chi_{11}^{5}\right\rangle$ & 4 & 1 & 8 & 37 & $\left\langle\chi_{5} \chi_{3} \chi_{7}^{3}, \chi_{7}^{3} \chi_{19}^{9}\right\rangle$ & 16 & 4 & 8 \\
\hline 19 & $\left\langle\chi_{5}, \chi_{4} \chi_{11}^{5}\right\rangle$ & 4 & 1 & 8 & 38 & $\left\langle\chi_{5} \chi_{3} \chi_{4} \psi_{8}, \chi_{4} \psi_{8} \chi_{19}^{9}\right\rangle$ & 16 & 4 & 8 \\
\hline
\end{tabular}

TABLE 7. $G(N / \mathbb{Q}) \simeq(4,2)$ with $G\left(N^{+} / \mathbb{Q}\right) \simeq \mathbb{Z} / 4 \mathbb{Z}$, $\exp (C l(N)) \leq 2, \exp (C l(K)) \leq 2$, and $\exp \left(C l\left(K^{\prime}\right)\right) \leq 2, K$ is associated with $\langle\chi\rangle$ and $K^{\prime}$ is associated with $\left\langle\chi^{2} \psi, \psi\right\rangle$.

\begin{tabular}{|c|c|c|c|c|c|c|c|c|c|}
\hline$N r$. & $X_{N}=\langle\chi, \psi\rangle$ & $h_{N}$ & $h_{K}$ & $h_{K^{\prime}}$ & $N r$. & $X_{N}=\langle\chi, \psi\rangle$ & $h_{N}$ & $h_{K}$ & $h_{K^{\prime}}$ \\
\hline 1 & $\left\langle\chi_{5}, \chi_{3}\right\rangle$ & 1 & 1 & 1 & 51 & $\left\langle\chi_{5} \psi_{8}, \chi_{7}^{3}\right\rangle$ & 4 & 2 & 1 \\
\hline 2 & $\left\langle\psi_{16} \chi_{4}, \chi_{4}\right\rangle$ & 1 & 1 & 1 & 52 & $\left\langle\chi_{5} \chi_{4} \chi_{7}^{3}, \chi_{4} \psi_{8}\right\rangle$ & 4 & 4 & 1 \\
\hline 3 & $\left\langle\chi_{5}, \chi_{4}\right\rangle$ & 1 & 1 & 1 & 53 & $\left\langle\chi_{5} \chi_{3} \chi_{19}^{9}, \chi_{3}\right\rangle$ & 4 & 8 & 1 \\
\hline 4 & $\left\langle\chi_{5}, \chi_{7}^{3}\right\rangle$ & 1 & 1 & 1 & 54 & $\left\langle\chi_{13}^{3}, \chi_{3} \psi_{8}\right\rangle$ & 4 & 1 & 4 \\
\hline 5 & $\left\langle\chi_{13}^{3}, \chi_{3}\right\rangle$ & 2 & 1 & 2 & 55 & $\left\langle\chi_{13}^{3} \psi_{8}, \chi_{3} \psi_{8}\right\rangle$ & 4 & 2 & 4 \\
\hline 6 & $\left\langle\chi_{5}, \psi_{8} \chi_{4}\right\rangle$ & 1 & 1 & 1 & 56 & $\left\langle\chi_{13}^{3} \chi_{3} \chi_{4}, \chi_{3} \psi_{8}\right\rangle$ & 16 & 8 & 4 \\
\hline 7 & $\left\langle\chi_{5} \psi_{8}, \chi_{4}\right\rangle$ & 2 & 2 & 1 & 57 & $\left\langle\psi_{16} \chi_{4}, \chi_{4} \chi_{3} \chi_{7}^{3}\right\rangle$ & 8 & 1 & 8 \\
\hline 8 & $\left\langle\chi_{5} \psi_{8}, \psi_{8} \chi_{4}\right\rangle$ & 2 & 2 & 1 & 58 & $\left\langle\psi_{16} \chi_{3}, \chi_{4} \chi_{3} \chi_{7}^{3}\right\rangle$ & 8 & 2 & 8 \\
\hline 9 & $\left\langle\psi_{16} \chi_{4}, \chi_{3}\right\rangle$ & 1 & 1 & 1 & 59 & $\left\langle\psi_{16} \chi_{7}^{3}, \chi_{3}\right\rangle$ & 4 & 4 & 1 \\
\hline 10 & $\left\langle\psi_{16} \chi_{3}, \chi_{4}\right\rangle$ & 2 & 2 & 1 & 60 & $\left\langle\psi_{16} \chi_{7}^{3}, \chi_{4} \chi_{3} \chi_{7}^{3}\right\rangle$ & 16 & 4 & 8 \\
\hline 11 & $\left\langle\psi_{16} \chi_{3}, \chi_{3}\right\rangle$ & 1 & 2 & 1 & 61 & $\left\langle\chi_{17}^{4} \chi_{4}, \chi_{4} \chi_{5}^{2}\right\rangle$ & 16 & 4 & 4 \\
\hline 12 & $\left\langle\chi_{13}^{3}, \chi_{4}\right\rangle$ & 1 & 1 & 1 & 62 & $\left\langle\chi_{5} \chi_{17}^{8}, \chi_{4}\right\rangle$ & 4 & 2 & 1 \\
\hline 13 & $\left\langle\chi_{5}, \chi_{11}^{5}\right\rangle$ & 2 & 1 & 2 & 63 & $\left\langle\chi_{17}^{4} \chi_{7}^{3}, \chi_{3}\right\rangle$ & 2 & 2 & 1 \\
\hline 14 & $\left\langle\chi_{5} \chi_{3} \chi_{4}, \chi_{3}\right\rangle$ & 2 & 4 & 1 & 64 & $\left\langle\chi_{17}^{4} \chi_{4}, \chi_{3} \psi_{8}\right\rangle$ & 16 & 4 & 4 \\
\hline 15 & $\left\langle\chi_{5} \chi_{3} \chi_{4}, \chi_{4}\right\rangle$ & 2 & 4 & 1 & 65 & $\left\langle\chi_{17}^{4} \chi_{4} \psi_{8}, \chi_{3}\right\rangle$ & 4 & 4 & 1 \\
\hline 16 & $\left\langle\psi_{16} \chi_{4}, \chi_{4} \chi_{5}^{2}\right\rangle$ & 2 & 1 & 2 & 66 & $\left\langle\chi_{17}^{4} \chi_{4} \psi_{8}, \chi_{3} \psi_{8}\right\rangle$ & 16 & 4 & 4 \\
\hline 17 & $\left\langle\psi_{16} \chi_{4} \chi_{5}^{2}, \chi_{4}\right\rangle$ & 2 & 2 & 1 & 67 & $\left\langle\chi_{5} \chi_{3} \chi_{4}, \chi_{7}^{3}\right\rangle$ & 8 & 4 & 1 \\
\hline 18 & $\left\langle\psi_{16} \chi_{4} \chi_{5}^{2}, \chi_{4} \chi_{5}^{2}\right\rangle$ & 2 & 2 & 2 & 68 & $\left\langle\chi_{5} \chi_{3} \chi_{7}^{3}, \chi_{4}\right\rangle$ & 8 & 4 & 1 \\
\hline 19 & $\left\langle\chi_{13}^{3}, \chi_{7}^{3}\right\rangle$ & 1 & 1 & 1 & 69 & $\left\langle\chi_{5} \chi_{4} \chi_{7}^{3}, \chi_{3}\right\rangle$ & 8 & 4 & 1 \\
\hline 20 & $\left\langle\chi_{13}^{3} \psi_{8}, \chi_{4}\right\rangle$ & 2 & 2 & 1 & 70 & $\left\langle\chi_{29}^{7} \chi_{5}^{2}, \chi_{3} \chi_{5}^{2}\right\rangle$ & 8 & 4 & 4 \\
\hline 21 & $\left\langle\chi_{5} \chi_{3} \chi_{7}^{3}, \chi_{3}\right\rangle$ & 2 & 4 & 1 & 71 & $\left\langle\chi_{5} \chi_{4} \chi_{11}^{5}, \chi_{4} \psi_{8}\right\rangle$ & 8 & 8 & 1 \\
\hline 22 & $\left\langle\chi_{5} \chi_{3} \chi_{7}^{3}, \chi_{7}^{3}\right\rangle$ & 2 & 4 & 1 & 72 & $\left\langle\chi_{5} \chi_{13}^{6}, \chi_{7}^{3}\right\rangle$ & 4 & 2 & 1 \\
\hline 23 & $\left\langle\psi_{16} \chi_{4}, \chi_{7}^{3}\right\rangle$ & 2 & 1 & 2 & 73 & $\left\langle\chi_{13}^{3} \chi_{5}^{2}, \chi_{7}^{3}\right\rangle$ & 4 & 2 & 1 \\
\hline 24 & $\left\langle\psi_{16} \chi_{7}^{3}, \chi_{4}\right\rangle$ & 4 & 4 & 1 & 74 & $\left\langle\chi_{41}^{10} \chi_{4}, \chi_{3}\right\rangle$ & 4 & 4 & 1 \\
\hline 25 & $\left\langle\chi_{5} \psi_{8}, \chi_{3}\right\rangle$ & 4 & 2 & 1 & 75 & $\left\langle\chi_{5} \chi_{13}^{6}, \chi_{4} \psi_{8}\right\rangle$ & 4 & 2 & 1 \\
\hline
\end{tabular}




\begin{tabular}{|c|c|c|c|c|c|c|c|c|c|}
\hline 26 & $\left\langle\chi_{5} \chi_{3} \chi_{4}, \chi_{4} \psi_{8}\right\rangle$ & 4 & 4 & 1 & 76 & $\left\langle\psi_{16} \chi_{3}, \chi_{11}^{5}\right\rangle$ & 2 & 2 & 1 \\
\hline 27 & $\left\langle\chi_{5} \chi_{3} \chi_{4} \psi_{8}, \chi_{3}\right\rangle$ & 2 & 4 & 1 & 77 & $\left\langle\chi_{37}^{9}, \chi_{3} \chi_{5}^{2}\right\rangle$ & 4 & 1 & 4 \\
\hline 28 & $\left\langle\chi_{5} \chi_{3} \chi_{4} \psi_{8}, \chi_{4}\right\rangle$ & 4 & 4 & 1 & 78 & $\left\langle\psi_{16} \chi_{4}, \chi_{5}^{2} \chi_{7}^{3}\right\rangle$ & 4 & 1 & 4 \\
\hline 29 & $\left\langle\chi_{5} \chi_{3} \chi_{4} \psi_{8}, \chi_{4} \psi_{8}\right\rangle$ & 2 & 4 & 1 & 79 & $\left\langle\psi_{16} \chi_{4} \chi_{5}^{2}, \chi_{5}^{2} \chi_{7}^{3}\right\rangle$ & 4 & 2 & 4 \\
\hline 30 & $\left\langle\chi_{5} \chi_{4} \chi_{7}^{3}, \chi_{4}\right\rangle$ & 2 & 4 & 1 & 80 & $\left\langle\psi_{16} \chi_{7}^{3}, \chi_{4} \chi_{5}^{2}\right\rangle$ & 8 & 4 & 2 \\
\hline 31 & $\left\langle\chi_{5} \chi_{4} \chi_{7}^{3}, \chi_{7}^{3}\right\rangle$ & 2 & 4 & 1 & 81 & $\left\langle\chi_{5} \chi_{29}^{14}, \chi_{4}\right\rangle$ & 8 & 4 & 1 \\
\hline 32 & $\left\langle\chi_{37}^{9}, \chi_{4}\right\rangle$ & 1 & 1 & 1 & 82 & $\left\langle\chi_{5} \chi_{17}^{8}, \chi_{7}^{3}\right\rangle$ & 4 & 2 & 1 \\
\hline 33 & $\left\langle\chi_{13}^{3} \chi_{3} \chi_{4}, \chi_{4}\right\rangle$ & 4 & 8 & 1 & 83 & $\left\langle\chi_{5} \chi_{17}^{8}, \chi_{4} \psi_{8}\right\rangle$ & 4 & 2 & 1 \\
\hline 34 & $\left\langle\chi_{5} \chi_{3} \chi_{11}^{5}, \chi_{3}\right\rangle$ & 4 & 8 & 1 & 84 & $\left\langle\chi_{17}^{4} \chi_{4}, \chi_{11}^{5}\right\rangle$ & 4 & 4 & 1 \\
\hline 35 & $\left\langle\psi_{16} \chi_{4}, \chi_{11}^{5}\right\rangle$ & 1 & 1 & 1 & 85 & $\left\langle\psi_{16} \chi_{4}, \chi_{3} \chi_{17}^{8}\right\rangle$ & 8 & 1 & 4 \\
\hline 36 & $\left\langle\chi_{13}^{3}, \chi_{3} \chi_{5}^{2}\right\rangle$ & 4 & 1 & 4 & 86 & $\left\langle\chi_{5} \chi_{3} \chi_{7}^{3}, \chi_{4} \psi_{8}\right\rangle$ & 8 & 4 & 1 \\
\hline 37 & $\left\langle\chi_{5} \chi_{13}^{6}, \chi_{3}\right\rangle$ & 4 & 2 & 1 & 87 & $\left\langle\chi_{5} \chi_{3} \chi_{4} \psi_{8}, \chi_{7}^{3}\right\rangle$ & 8 & 4 & 1 \\
\hline 38 & $\left\langle\chi_{13}^{3} \chi_{5}^{2}, \chi_{3} \chi_{5}^{2}\right\rangle$ & 4 & 2 & 4 & 88 & $\left\langle\chi_{73}^{18} \chi_{3}, \chi_{4}\right\rangle$ & 32 & 4 & 2 \\
\hline 39 & $\left\langle\chi_{29}^{7}, \chi_{7}^{3}\right\rangle$ & 2 & 1 & 2 & 89 & $\left\langle\psi_{16} \chi_{4} \chi_{5}^{2}, \chi_{11}^{5}\right\rangle$ & 4 & 2 & 1 \\
\hline 40 & $\left\langle\chi_{17}^{4} \chi_{4}, \chi_{3}\right\rangle$ & 4 & 4 & 1 & 90 & $\left\langle\chi_{13}^{3} \chi_{17}^{8}, \chi_{4}\right\rangle$ & 8 & 4 & 1 \\
\hline 41 & $\left\langle\chi_{5} \chi_{4} \chi_{11}^{5}, \chi_{4}\right\rangle$ & 4 & 8 & 1 & 91 & $\left\langle\chi_{5} \chi_{29}^{14}, \chi_{7}^{3}\right\rangle$ & 16 & 4 & 1 \\
\hline 42 & $\left\langle\chi_{29}^{7}, \chi_{4} \psi_{8}\right\rangle$ & 1 & 1 & 1 & 92 & $\left\langle\chi_{17}^{4} \chi_{3} \chi_{5}^{2}, \chi_{4} \chi_{5}^{2}\right\rangle$ & 16 & 4 & 4 \\
\hline 43 & $\left\langle\psi_{16} \chi_{3}, \chi_{4} \chi_{5}^{2}\right\rangle$ & 4 & 2 & 2 & 93 & $\left\langle\chi_{5} \chi_{3} \chi_{11}^{5}, \chi_{7}^{3}\right\rangle$ & 16 & 8 & 1 \\
\hline 44 & $\left\langle\psi_{16} \chi_{4} \chi_{5}^{2}, \chi_{3}\right\rangle$ & 4 & 2 & 1 & 94 & $\left\langle\psi_{16} \chi_{7}^{3}, \chi_{11}^{5}\right\rangle$ & 4 & 4 & 1 \\
\hline 45 & $\left\langle\chi_{5} \chi_{17}^{8}, \chi_{3}\right\rangle$ & 4 & 2 & 1 & 95 & $\left\langle\chi_{17}^{4} \chi_{7}^{3}, \chi_{11}^{5}\right\rangle$ & 2 & 2 & 1 \\
\hline 46 & $\left\langle\chi_{17}^{4} \chi_{3} \chi_{5}^{2}, \chi_{3}\right\rangle$ & 4 & 4 & 1 & 96 & $\left\langle\chi_{5} \chi_{3} \chi_{11}^{5}, \chi_{4} \psi_{8}\right\rangle$ & 16 & 8 & 1 \\
\hline 47 & $\left\langle\chi_{37}^{9}, \chi_{7}^{3}\right\rangle$ & 2 & 1 & 2 & 97 & $\left\langle\chi_{17}^{4} \psi_{8} \chi_{4}, \chi_{11}^{5}\right\rangle$ & 4 & 4 & 1 \\
\hline 48 & $\left\langle\chi_{5} \chi_{13}^{6}, \chi_{4}\right\rangle$ & 4 & 2 & 1 & 98 & $\left\langle\chi_{5} \chi_{4} \chi_{11}^{5}, \chi_{7}^{3}\right\rangle$ & 16 & 8 & 1 \\
\hline 49 & $\left\langle\chi_{13}^{3} \chi_{5}^{2}, \chi_{4}\right\rangle$ & 4 & 2 & 1 & 99 & $\left\langle\chi_{5} \chi_{3} \chi_{19}^{9}, \chi_{4} \psi_{8}\right\rangle$ & 16 & 8 & 1 \\
\hline 50 & $\left\langle\chi_{13}^{3} \chi_{3} \chi_{7}^{3}, \chi_{7}^{3}\right\rangle$ & 4 & 8 & 1 & 100 & $\left\langle\chi_{17}^{4} \chi_{3} \chi_{5}^{2}, \chi_{11}^{5}\right\rangle$ & 8 & 4 & 1 \\
\hline
\end{tabular}

TABLE 8. $G(N / \mathbb{Q}) \simeq(8,2)$ with $G\left(N^{+} / \mathbb{Q}\right) \simeq(4,2), \exp (C l(N)) \leq$ $2, \exp (C l(K)) \leq 2$, and $\exp \left(C l\left(K^{\prime}\right)\right) \leq 2, K$ is associated with $\langle\chi\rangle$ and $K^{\prime}$ is associated with $\langle\chi \psi\rangle$.

\begin{tabular}{|c|c|c|c|c|}
\hline$N r$. & $X_{N}=\langle\chi, \psi\rangle$ & $h_{N}$ & $h_{K}$ & $h_{K^{\prime}}$ \\
\hline 1 & $\left\langle\chi_{17}^{2} \chi_{4}, \chi_{4} \chi_{3}\right\rangle$ & 4 & 4 & 2 \\
\hline
\end{tabular}

TABLE 9. $G(N / \mathbb{Q}) \simeq(8,2)$ with $G\left(N^{+} / \mathbb{Q}\right) \simeq \mathbb{Z} / 8 \mathbb{Z}$, $\exp (C l(N)) \leq 2, \exp (C l(K)) \leq 2$, and $\exp \left(C l\left(K^{\prime}\right)\right) \leq 2, K$ is associated with $\langle\chi\rangle$ and $K^{\prime}$ is associated with $\left\langle\chi^{2} \psi, \psi\right\rangle$.

\begin{tabular}{|c|c|c|c|c|}
\hline$N r$. & $X_{N}=\langle\chi, \psi\rangle$ & $h_{N}$ & $h_{K}$ & $h_{K^{\prime}}$ \\
\hline 1 & $\left\langle\psi_{32} \chi_{4}, \chi_{4}\right\rangle$ & 1 & 1 & 1 \\
\hline 2 & $\left\langle\psi_{32} \chi_{4}, \chi_{3}\right\rangle$ & 1 & 1 & 1 \\
\hline 3 & $\left\langle\psi_{32} \chi_{4}, \chi_{5}^{2} \chi_{4}\right\rangle$ & 2 & 1 & 2 \\
\hline
\end{tabular}


TABLE 10. $G(N / \mathbb{Q}) \simeq(4,4)$ with $\exp (C l(N)) \leq 2, \exp (C l(K)) \leq$ 2 , and $\exp \left(C l\left(K^{\prime}\right)\right) \leq 2, K$ is associated with $\left\langle\chi^{2}, \psi\right\rangle$ and $K^{\prime}$ is associated with $\left\langle\chi, \psi^{2}\right\rangle$.

\begin{tabular}{|c|c|c|c|c|}
\hline$N r$. & $X_{N}=\langle\chi, \psi\rangle$ & $h_{N}$ & $h_{K}$ & $h_{K^{\prime}}$ \\
\hline 1 & $\left\langle\chi_{5}, \psi_{16} \chi_{4}\right\rangle$ & 1 & 1 & 1 \\
\hline 2 & $\left\langle\chi_{5}, \chi_{13}^{3}\right\rangle$ & 1 & 1 & 1 \\
\hline 3 & $\left\langle\chi_{5} \chi_{3} \chi_{4}, \psi_{16} \chi_{4}\right\rangle$ & 8 & 1 & 8 \\
\hline
\end{tabular}

\section{REFERENCES}

[AD] Francesco Amoroso and Roberto Dvornicich, Lower bounds for the height and size of the ideal class group in CM-Fields, Monatsh. Math. 138 (2003), no. 2, 85-94. MR.1963737 (2004e:11124)

[AK] Jeoung-Hwan Ahn and Soun-Hi Kwon, The class groups of the imaginary abelian number fields with Galois group $(\mathbb{Z} / 2 \mathbb{Z})^{n}$, Bull. Austral. Math. Soc. 70 (2004), no. 2, 267-277. MR.2094294 (2005e:11142)

[BK] D. W. Boyd and H. Kisilevsky, On the exponent of the ideal class groups of complex quadratic fields, Proc. Amer. Math. Soc. 31 (1972), 433-436. MR0289454 (44:6644)

[CF] J. W. S. Cassels and A. Fröhich, Algebraic number theory, Academic Press, London and New York, 1967. MR0215665 (35:6500)

[CH] P. E. Conner and J. Hurrelbrink, Class number parity, Series in Pure Mathematics Volume 8, World Scientific, 1988. MR963648 (90f:11092)

[Che] C. Chevalley, Sur la théorie du corps de classes dans les corps finis et les corps locaux, Jour. of the Fac. of Sc., Tokyo, vol. II, Part 9 (1933).

[Cho] S. Chowla, An extension of Heilbronn's class number theorem, Quart. J. Math. Oxford Ser. (2) 5 (1934), 304-307.

[E] A. G. Earnest, Exponents of the class groups of imaginary abelian number fields, Bull. Austral. Math. Soc. 35 (1987), no. 2, 231-245. MR878434 (88d:11103)

[G] G. Gras, Sur les l-classes d'ideaux dans les extensions cycliques relatives de degré premier l, Ann. Inst. Fourier, Grenoble 23.3 (1973), 1-48. MR0360519 (50:12967)

[Ha] H. Hasse, Über die Klassenzahl abelscher Zahlkörper, Academie-Verlag, Berlin, 1952. Reprinted with an introduction by J. Martinet, Springer-Verlag, Berlin (1985).

[HB] D. R. Heath-Brown, Imaginary quadratic fields with class group exponent 5 , Forum Math. 20 (2008), 275-283. MR.2394923 (2009b:11194)

[HH] K. Horie and M. Horie, CM-fields and exponents of their ideal class groups, Acta Arith. 55 (1990), no. 2, 157-170. MR.1061636 (91k:11098)

[KT] M. Daberkow, C. Fieker, J. Klüners, M. Phost, K. Roegner and K. Wildanger, KANT V4, in J. Symbolic Comp. 24 (1997), 267-283. MR1484479 (99g:11150)

[LO] S. Louboutin and R. Okazaki, Exponents of the ideal class groups of CM number fields, Math. Z. 243 (2003), no. 1, 155-159. MR.1953054 (2003j:11136)

[Lou90] S. Louboutin, Minoration (sous l'hypothése de Riemann généralisée) des nombres de classes des corps quadratiques imaginaires. Application, C. R. Acad. Sci. Paris 310 (1990), 795-800. MR.1058499 (91e:11126)

[Lou95] S. Louboutin, Determination of all nonquadratic imaginary cyclic number fields of 2power degrees with ideal class groups of exponents $\leq 2$, Math. Comp. 64 (1995), no. 209, 323-340. MR 1248972 (95c:11124)

[Lou97] S. Louboutin, CM-fields with cyclic ideal class groups of 2-power orders, J. Number Theory 67 (1997), no. 1, 1-10. MR1485424(98h:11139)

[Lou01] S. Louboutin, Computation of $L(0, \chi)$ and of relative class numbers of $C M$-fields, Nagoya Math. J., Vol. 161(2001), 171-191. MR.1820217 (2002e:11152)

[Mas] J. M. Masley, Class numbers of real cyclic number fields with small conductor, Compos. Math. 37 (1978), 297-339. MR511747 (80e:12005)

[N] J. Neukirch, Class field theory, Grundlehren der Matematischen Wissenschaften 280, Springer-Verlag, Berlin Heidelberg, 1986. MR819231(87i:11005) 
[P] B. Allombert, K. Belabas, H. Cohen, X. Robolot, and I. Zakharevich, PARI/GP, version 2.3.4, Bordeaux, 2008, http://pari.math.u-bordeaux.fr/.

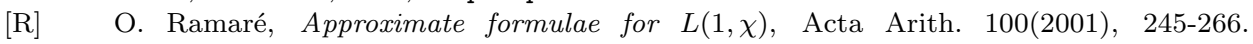
MR 1865385 (2002k:11144)

[Wa] L. Washington, Introduction to cyclotomic fields, 2nd ed., Graduate texts in Mathematics, vol. 83, Springer-Verlag, New York, 1997. MR1421575 (97h:11130)

[We] P. J. Weinberger, Exponents of the class groups of complex quadratic fields, Acta Arith., 22 (1973), 117-124. MR0313221 (47:1776)

Department of Mathematics Education, Korea University, 136-701, Seoul, Korea

E-mail address: jh-ahn@korea.ac.kr

Department of Mathematics Education, Korea University, 136-701, Seoul, Korea

E-mail address: sounhikwon@korea.ac.kr 